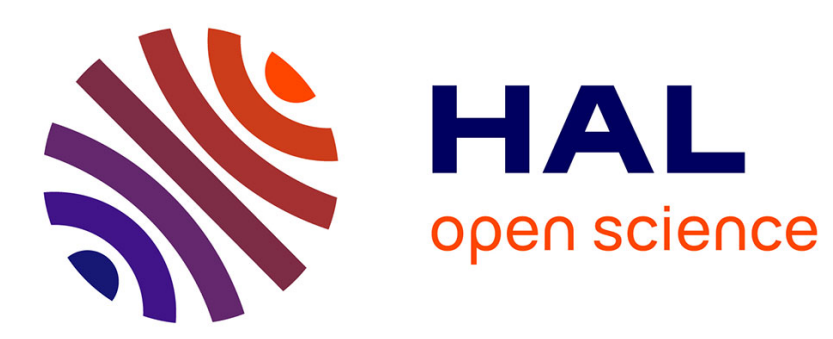

\title{
On a model of layered piezoelectric beams including transverse stress effect
}

\author{
Corrado Maurini, Joël Pouget, Francesco Dell'Isola
}

\section{To cite this version:}

Corrado Maurini, Joël Pouget, Francesco Dell'Isola. On a model of layered piezoelectric beams including transverse stress effect. International Journal of Solids and Structures, 2004, pp.30. hal-00503237

\section{HAL Id: hal-00503237 \\ https://hal.science/hal-00503237}

Submitted on 19 Jul 2010

HAL is a multi-disciplinary open access archive for the deposit and dissemination of scientific research documents, whether they are published or not. The documents may come from teaching and research institutions in France or abroad, or from public or private research centers.
L'archive ouverte pluridisciplinaire HAL, est destinée au dépôt et à la diffusion de documents scientifiques de niveau recherche, publiés ou non, émanant des établissements d'enseignement et de recherche français ou étrangers, des laboratoires publics ou privés. 


\title{
On a model of layered piezoelectric beams including transverse stress effect
}

\author{
Corrado Maurini ${ }^{\mathrm{a}, \mathrm{b}}$, Joël Pouget ${ }^{\mathrm{a}, *}$, Francesco dell'Isola ${ }^{\mathrm{c}}$ \\ ${ }^{a}$ Laboratoire d'Etudes Mécaniques des Assemblages (CNRS-FRE 2481), Université de Versailles/Saint-Quentin-en-Yvelines, \\ Batiment Descartes, 45 Avenue des Etats-Unis, 78035 Versailles, France \\ b Dipartimento di Meccanica e Aeronautica, Università di Roma, "La Sapienza", via Eudossiana 18, 00184 Rome, Italy \\ c Dipartimento di Ingegneria Strutturale e Geotecnica, Università di Roma "La Sapienza", via Eudossiana 18, 00184 Rome, Italy.
}

Received 2 October 2003; received in revised form 1 March 2004

Available online 15 April 2004

\begin{abstract}
In this paper a Euler-Bernoulli-like model of layered piezoelectric beams is presented. It describes more accurately than the others already presented in the literature both transverse (Poisson and piezoelectrically induced) cross-sectional deformations and through-the-thickness variations of the electric field and electric displacement. A deductive approach based on a mixed variational formulation is adopted and distributions of deformation, stress, electric field and electric displacement are simultaneously prescribed. The attention is focused on the choice of the most fitting assumptions to recover complex 3D cross-sectional field distributions. In particular, transverse interactions between different layers are taken into account by enforcing specific conditions on transverse stress through the Lagrange multipliers method. The estimate of electromechanical beam constitutive coefficients is discussed and comparison with standard modelling approaches, which assume either vanishing transverse stresses or vanishing transverse strains, is emphasized. For a sandwich piezoelectric beam and for a two-layer beam, expressions of the beam constitutive coefficients are provided and the main features of the proposed model are highlighted by presenting the through-thethickness distribution of the 3D state fields associated to beam-axis deformations and applied voltage. As a main peculiarity, the proposed beam model is able to coherently estimate the equivalent piezoelectric capacitance also when the thickness of elastic and piezoelectric layers is comparable.
\end{abstract}

(C) 2004 Elsevier Ltd. All rights reserved.

Keywords: Euler-Bernoulli beam; Piezocomposites; Sandwich materials; Mixed variational formulation; Laminate; Piezoelectric capacitance; Layered beams

\section{Introduction}

Composite materials made of active piezoelectric layers have been widely used for their sensor and actuator functions and research in this area opens many applications in the domain of adaptive structures

\footnotetext{
${ }^{*}$ Corresponding author. Tel.: +33-1-39-25-42-09; fax: +33-1-39-25-30-15.

E-mail address: joel.pouget@meca.uvsq.fr (J. Pouget).
} 
and structural control. Piezoelectric materials, and especially piezoelectric beam composites, are excellent candidates for designing adaptive devices or smart materials, and numerous applications in the domain of advanced technology have been proposed, running from aerospace or automotive (shape control of space antennas or telescopes, vibration control of helicopter blades, noise reduction, etc.) to micropositioners or microactuators (in medical apparatuses, measurement devices, robotics, etc.), as reported by Rao and Sunar (1994), Tani et al. (1998), Chee et al. (1998), and Chopra (2002). The high performance of piezoelectric composites becomes a requirement in the design of vibration control systems. Especially, piezoelectric elements can be used as components for passive damping systems, thereby avoiding complex control and feedback strategies (see e.g. Anderson and Hagood, 1994; Hagood and Von Flotow, 1991).

The present work attempts to present a consistent and efficient deductive approach to piezoelectric laminated beams. Slender beam-shaped structures incorporating piezoelectric materials are often used in engineering applications (robotic arms, airplane wings, rotor blades, etc.). One dimensional modelling allows for a thumbnail description of their static and dynamic behavior. As the related one-dimensional boundary-value problems usually can be solved analytically, it provides useful tools for structural design and control applications. When deductive approaches are followed, one-dimensional models of piezoelectric laminated beams are obtained from a three-dimensional formulation by assuming a priori distribution of mechanical and electrical state fields. In such a micro-macro identification procedure, the beam balance equations are found and the corresponding constitutive relations are expressed as functions of the material and geometrical data at the three-dimensional level. The so-called induced strain models seem to supply the simplest description of the actuation mechanism in laminated piezoelectric beams (see e.g. Alzahrani and Alghamdi, 2003). They can be classified into those assuming a simple extensional strain within the piezoelectric elements (uniform strain model, see Park et al., 1996; Hong and Chopra, 1999), and those accounting for extensional and bending deformations of the piezoelectric elements as prescribed by standard Bernoulli-Euler beam theory (see Crawley and de Luis, 1987; Crawley and Anderson, 1990). A number of more refined and consistent approaches to piezoelectric laminated beam have been proposed (reviews can be found in Chopra, 2002; Saravanos and Heyliger, 1999; Gopinathan et al., 2000). They include the description of shear deformations, effects of bonding layers, full two-fold electromechanical coupling (i.e. including both of direct and inverse piezoelectric effects), etc. Such models, although onedimensional, can become very complex by including several variables of microstructures to mimic the threedimensional behavior. However, as underlined also by Beckert and Pfundtner (2002), most of these models are based on assumptions on the stress state that are too restrictive. For example, in unshearable models, it is usually assumed (see e.g. Alzahrani and Alghamdi, 2003) that the stress tensor is in the form (refer to Fig. 1 for notation and beam cross-sectional geometry)

$$
\mathbf{T}=T_{11}\left(\mathbf{e}_{1} \otimes \mathbf{e}_{1}\right) .
$$

Due to this assumption, transverse interactions (in the width $\mathbf{e}_{2}$ direction) between different layers are neglected and each layer is left free to deform in the transverse direction independently of the others, without respecting the bonding condition. The consequence of this assumption is reflected in an incorrect estimate of the one-dimensional constitutive equations. This issue is particularly relevant in layered beams including piezoelectric layers polarized along the thickness ( $\mathbf{e}_{3}$ direction). Indeed, when a potential difference is applied between the electrodes of a piezoelectric layer, it naturally tends to isotropically extend (or shrink) in the plane orthogonal to the polarization axis $\left(\mathbf{e}_{1}-\mathbf{e}_{2}\right.$ plane). This behavior is in competition with the Poisson effect in the elastic layer: when one tries to extend an elastic layer in one direction (say $\mathbf{e}_{1}$ ), shrinking in the other direction (say $\mathbf{e}_{2}$ ) is typically induced. When piezoelectric and elastic layers are bonded together to form a laminated piezoelectric beam, their contrasting behavior is conciliated by the appearance of non-negligible normal stresses both in the axial direction $\left(T_{11}\right.$ stress $)$ and transverse direction ( $T_{22}$ stress). In Beckert and Pfundtner (2002) this problem is discussed: assumption (1) is improved by including transverse $\left(T_{22}\right)$ stresses, in order to describe more accurately the complex interactions between 


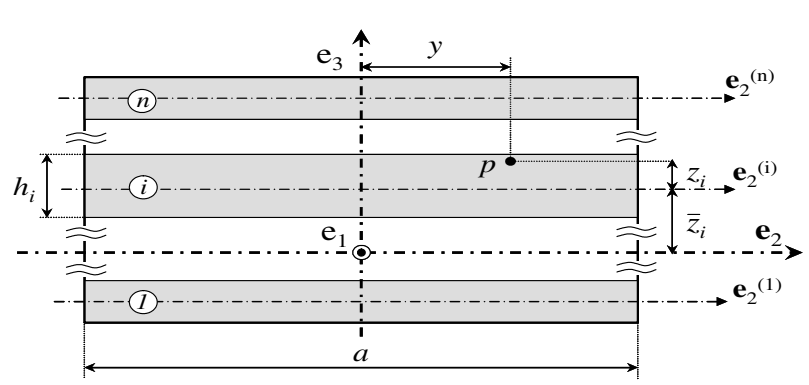

Fig. 1. Generic layered piezoelectric beam: cross-section.

different layers in composite piezoelectric laminates. However, only actuation functions are considered and no attention is paid to the determination of purely electrical properties such as the equivalent piezoelectric capacitance. Its incorrect estimate can be misleading when piezoelectric elements are used as transducers, as in passive vibration control devices similar to the electric vibration absorber studied by Hagood and Von Flotow (1991). Indeed, in this case, an optimal design of the electric controller is strongly influenced by mechanical, coupling, and electrical properties of the layered piezoelectric beam.

The aim of the present work is to discuss the consequences of transverse interactions between different layers in piezoelectric laminates and to propose a novel efficient model including both of sensory and actuation effects. The model thus proposed is based on (i) equivalent single-layer Bernoulli-Euler kinematics (linear strain distribution along the thickness direction of the beam); (ii) layerwise linear distribution of mechanical stress with non-vanishing transverse normal stress (i.e. $T_{22}$ ); (iii) layerwise linear distribution of electric potential; (iv) layerwise constant distribution of the electric displacement. In particular, we try to refine the description achieved by simplest modelling approaches without adding additional degrees of freedom to describe more complex phenomena such as shear effects, bonding-layer effects, higher-order distribution of electric field and displacement, etc. As a main peculiarity, the proposed model accounts for transverse interactions between different layers by including a non-vanishing distribution of normal transverse stresses. These additional fields are found among those verifying further integral force balance laws, corresponding to transverse projections of force and moment balance laws for each beam crosssection. These conditions will be referred to as weak conditions on transverse stress. Such a model, characterized by assuming null transverse stress resultants (NSR model), is compared to standard models assuming either pointwise null transverse stress (NS model), or null transverse sectional deformations (ND model). One of the purposes of the present work is to examine and discuss how the newly introduced stress field $T_{22}$ and the associated weak conditions on it enhance the estimate of the electromechanical constitutive properties of piezoelectric laminated beams.

The proposed beam model is deduced from the three-dimensional description by assuming at the same time hypotheses on strain and stress, on electric field and electric displacement. To this end, a mixed variational formulation of the Hellinger-Prange-Reissner type is adopted (see $\mathrm{He}, 2000$; Teresi and Tiero, 1997; Washizu, 1982). Moreover, Lagrange multipliers are introduced to impose in the variational formulation the different conditions on transverse stress.

The prerequisites for three-dimensional piezoelectricity (equations of motion, boundary conditions and constitutive equations) and the associated mixed variational formulation are briefly recalled in the next section. In Section 3, beam governing equations are deduced from the mixed variational formulation by assuming peculiar distributions of three-dimensional state fields. In particular, governing equations for models assuming either null transverse stress resultants (NSR), pointwise null transverse stress (NS), or null transverse deformations (ND) are derived. In Section 4, comparisons between the different models are made and the main properties of the NSR model are underlined. To this end, particular cross-sectional 
configurations are considered: a three-layered sandwich piezoelectric beam with in-phase parallel connected piezoelectric layers; a three-layered sandwich piezoelectric beam with counter-phase parallel connected piezoelectric layers; and a two-layered piezoelectric beam. For the different conditions on the transverse stress, analytical formulas and numerical illustrations of beam constitutive coefficients are provided. Their dependence on material properties and the relative thickness ratio between different layers are analyzed. The through-the-thickness distributions of stress, strain, electric field and electric displacement are also presented. Lastly, Section 5 is devoted to the summary and discussion of the most pertinent results and extensions of the present beam model are suggested.

\section{Mixed variational formulation of 3D linear piezoelectricity}

In this section, the basic equations of three-dimensional linear piezoelectricity are recalled and a mixed variational formulation for the associated boundary value problem is proposed. Moreover, the application of the mixed variational formulation to structural modelling is briefly discussed.

\subsection{Equations of linearly piezoelectricity}

Consider a piezoelectric body that is identified by means of its reference configuration $\mathscr{B}$. In the quasielectrostatic approximation, the actual kinematical state of the system is described by the mechanical displacement field $\mathbf{u}$ and by the electric potential $\varphi$. Let us denote by $\partial \mathscr{B}$ the boundary of $\mathscr{B}$, by $\partial_{\mathbf{u}} \mathscr{B}$ and $\partial_{\mathbf{f}} \mathscr{B}$ the parts of $\partial \mathscr{B}$ where the displacements $\mathbf{u}_{0}$ and the tractions $\mathbf{f}_{0}$ are imposed, by $\partial_{\varphi} \mathscr{B}$ and $\partial_{q} \mathscr{B}$ the parts on which the electric potential $\varphi_{0}$ and the charge density $q_{0}$ are imposed $\left(\partial_{\mathbf{u}} \mathscr{B} \cup \partial_{\mathbf{f}} \mathscr{B}=\partial_{\varphi} \mathscr{B} \cup \partial_{q} \mathscr{B}=\partial \mathscr{B}\right.$ and $\left.\partial_{\mathbf{u}} \mathscr{B} \cap \partial_{\mathbf{f}} \mathscr{B}=\partial_{\varphi} \mathscr{B} \cap \partial_{q} \mathscr{B}=\emptyset\right)$. Let $\mathbf{S}$ be the linearized strain tensor, $\mathbf{T}$ the Cauchy stress tensor, $\mathbf{D}$ the electric displacement vector, and $\mathbf{E}$ the electric field vector inside the piezoelectric body.

In the quasi-electrostatic approximation, a strong formulation of the equations of linearly piezoelectricity is provided by the following equations (see e.g. Eringen and Maugin, 1990)

$$
\begin{aligned}
& \text { force balance : }\left\{\begin{array}{l}
\nabla \cdot \mathbf{T}+\mathbf{b}=\mathbf{0} \\
\nabla \cdot \mathbf{D}=0
\end{array} \text { on } \mathscr{B},\right. \\
& \text { kinematic compatibility : }\left\{\begin{array}{l}
\mathbf{S}=\operatorname{Sym}(\nabla \mathbf{u}) \\
\mathbf{E}=-\nabla \varphi
\end{array} \text { on } \mathscr{B},\right. \\
& \text { essential boundary conditions : } \begin{cases}\mathbf{u}=\mathbf{u}_{0} & \text { on } \partial_{\mathbf{u}} \mathscr{B}, \\
\varphi=\varphi_{0} & \text { on } \partial_{\varphi} \mathscr{B},\end{cases} \\
& \text { natural boundary conditions : } \begin{cases}\mathbf{T n}=\mathbf{f}_{0} & \text { on } \partial_{\mathbf{f}} \mathscr{B}, \\
\mathbf{D n}=q_{0} & \text { on } \partial_{q} \mathscr{B},\end{cases} \\
& \text { constitutive equations : }\left\{\begin{array}{l}
\mathbf{S}=\left.\frac{\partial \mathscr{F}}{\partial \mathbf{T}}\right|_{\mathbf{D}}=\mathfrak{s}^{\mathrm{D}} \mathbf{T}+\mathfrak{g}^{t} \mathbf{D} \\
\mathbf{E}=-\left.\frac{\partial \mathscr{F}}{\partial \mathbf{D}}\right|_{\mathbf{T}}=-\mathfrak{g} \mathbf{T}+\beta^{\mathrm{T}} \mathbf{D}
\end{array} \quad \text { on } \mathscr{B} .\right.
\end{aligned}
$$

In the equations above $\mathbf{n}$ denotes the external unit normal to the boundary $\partial \mathscr{B}, \mathbf{b}$ is an external force per unit volume applied on $\mathscr{B}$ (and eventually including inertial forces), $\nabla(\cdot)$ denotes the spatial gradient with respect to the reference position, $\nabla \cdot(\cdot)$ the corresponding divergence operator, and $\operatorname{Sym}(\cdot)$ extracts the symmetric part of a second order tensor. The constitutive equations are given in the so-called T-D form through the free energy density function for linear piezoelectricity (see Ikeda, 1990) 


$$
\mathscr{F}(\mathbf{T}, \mathbf{D})=\frac{1}{2} \mathfrak{s}^{\mathrm{D}} \mathbf{T} \cdot \mathbf{T}-\frac{1}{2} \beta^{\mathrm{T}} \mathbf{D} \cdot \mathbf{D}+\mathfrak{g} \mathbf{T} \cdot \mathbf{D}
$$

where $\mathfrak{s}^{\mathrm{D}}$ is the mechanical compliance fourth order tensor for null electric displacement, $\mathfrak{g}$ is the piezoelectric coupling third order tensor and $\beta^{\mathrm{T}}$ is a second order tensor modelling the inverse of the electric permittivity for null mechanical stress. For a transversely isotropic piezoelectric material, the tensors appearing in the constitutive equations are represented in the classical Voigt notation by means of the following matrices

$$
\begin{aligned}
& \beta^{\mathrm{T}}=\left[\begin{array}{ccc}
\beta_{11}^{\mathrm{T}} & 0 & 0 \\
0 & \beta_{11}^{\mathrm{T}} & 0 \\
0 & 0 & \beta_{33}^{\mathrm{T}}
\end{array}\right], \\
& \mathrm{s}^{\mathrm{D}}=\left[\begin{array}{cccccc}
s_{11}^{\mathrm{D}} & s_{12}^{\mathrm{D}} & s_{13}^{\mathrm{D}} & 0 & 0 & 0 \\
s_{12}^{\mathrm{D}} & s_{11}^{\mathrm{D}} & s_{13}^{\mathrm{D}} & 0 & 0 & 0 \\
s_{13}^{\mathrm{D}} & s_{13}^{\mathrm{D}} & s_{33}^{\mathrm{D}} & 0 & 0 & 0 \\
0 & 0 & 0 & s_{44}^{\mathrm{D}} & 0 & 0 \\
0 & 0 & 0 & 0 & s_{44}^{\mathrm{D}} & 0 \\
0 & 0 & 0 & 0 & 0 & 2\left(s_{11}^{\mathrm{D}}-s_{12}^{\mathrm{D}}\right)
\end{array}\right], \\
& g=\left[\begin{array}{cccccc}
0 & 0 & 0 & 0 & g_{15} & 0 \\
0 & 0 & 0 & g_{15} & 0 & 0 \\
g_{31} & g_{31} & g_{33} & 0 & 0 & 0
\end{array}\right] .
\end{aligned}
$$

\subsection{Mixed variational formulation and reduced models}

Let us define the affine space $\mathscr{V}_{\mathbf{u}}$ of kinematically admissible displacement and strain tensors and the space $\mathscr{V}_{\varphi}$ of admissible electric potential and electric field vectors as follows:

$$
\begin{aligned}
& \mathscr{V}_{\mathbf{u}} \equiv\left\{(\mathbf{u}, \mathbf{S}): \mathbf{u}=\mathbf{u}_{0} \text { on } \partial_{\mathbf{u}} \mathscr{B} \text { and } \mathbf{S}=\operatorname{Sym}(\nabla \mathbf{u}) \text { on } \mathscr{B}\right\}, \\
& \mathscr{V}_{\varphi} \equiv\left\{(\varphi, \mathbf{E}): \varphi=\varphi_{0} \text { on } \partial_{\varphi} \mathscr{B} \text { and } \mathbf{E}=-\nabla \varphi \text { on } \mathscr{B}\right\} .
\end{aligned}
$$

Moreover, let us denote by $\mathscr{V}_{\mathbf{T}}$ and $\mathscr{V}_{\mathbf{D}}$ the vector spaces of symmetric stress tensor fields and of electric displacements vector fields defined on $\mathscr{B}$.

Let us consider the following functional (Hellinger-Prange-Reissner functional for piezoelectricity (see Yang and Batra, 1995, Batra and Vidoli, 2002))

$$
\Theta[(\mathbf{u}, \mathbf{S}),(\boldsymbol{\varphi}, E), \mathbf{T}, \mathbf{D}]=\int_{\mathscr{B}}(\mathscr{F}(\mathbf{T}, \mathbf{D})-\mathbf{T} \cdot \mathbf{S}-\mathbf{D} \cdot \mathbf{E}+\mathbf{b} \cdot \mathbf{u}) \mathrm{d} \mathscr{B}+\int_{\partial_{\mathbf{f}} \mathscr{B}} \mathbf{f}_{0} \cdot \mathbf{u} \mathrm{d} \mathscr{S}-\int_{\partial_{q} \mathscr{B}} q_{0} \varphi \mathrm{d} \mathscr{S}
$$

defined over the space

$$
\mathscr{V}=\mathscr{V}_{\mathbf{u}} \times \mathscr{V}_{\varphi} \times \mathscr{V}_{\mathbf{T}} \times \mathscr{V}_{\mathbf{D}}
$$

Under suitable regularity conditions, it can be verified that the solution to the problem of linear piezoelectricity (2) is characterized by rendering stationary the Hellinger-Prange-Reissner functional (6) over $\mathscr{V}$. In particular, the equilibrium equations (2a) and the natural boundary conditions (2d) are found by imposing vanishing first variations of functional (6) with respect to $\mathbf{u}$ and $\varphi$, and the constitutive equations 
(2e) with respect to $\mathbf{T}$ and $\mathbf{D}$. On the other hand, the compatibility conditions (2b) and the essential boundary conditions (2c) are included in the definitions (5a) and (5b) of the functional spaces $\mathscr{V}_{\mathbf{u}}$ and $\mathscr{V}_{\varphi}$.

In a model derived by a mixed variational formulation, it is often useful to distinguish between the generalized deformations (S, E) obtained from the generalized displacements $(\mathbf{u}, \varphi)$ through the kinematic compatibility (2b) and the generalized deformations $(\overline{\mathbf{S}}, \overline{\mathbf{E}})$ obtained from the generalized stresses $(\mathbf{T}, \mathbf{D})$ though the constitutive equations (2e). Here and henceforth, the latter will be differentiated from the former by a superscripted bar.

By following a direct approach, reduced models of a given refined one are deduced by assuming specific a priori "types" of state fields. The accuracy of the description supplied by the reduced model relies on how judiciously the aforementioned "types" are chosen. By following the mixed variational formulation above, restrictive hypotheses on the generalized displacement $(\mathbf{u}, \varphi)$, as well as on generalized stresses $(\mathbf{T}, \mathbf{D})$, are straightforwardly included in the model. These hypotheses are introduced in the variational principle as constraints on the space $\mathscr{V}$ on which the variations of the mixed functional must be taken. In particular, when these constraints are linear, the constrained variational problem can be formulated as the search for stationary points of the given functional on a linear subspace $\mathscr{W}$ of $\mathscr{V}$. Alternatively, the constraints on the admissible state fields can be imposed in the variational formulation by the Lagrange multiplier method. The latter approach is particularly useful when the constraints naturally appear in an implicit form. Moreover, the physical interpretation of the Lagrange multipliers furnishes a deeper understanding of the problem.

\section{Beam models}

In this section, the three-dimensional mixed variational formulation is used to deduce beam models of slender piezoelectric laminates by assuming specific hypotheses on the cross-sectional distribution of the electromechanical state fields. In particular, by focusing on Euler-Bernoulli-like models, we investigate how assumptions on cross-sectional strains and stresses influence the estimate of the beam constitutive coefficients. Indeed, standard beam models (see e.g. Crawley and de Luis, 1987; Crawley and Anderson, 1990; Park et al., 1996) assume very drastic hypotheses about the distribution of transverse normal stress or strain, which although working well for mono-layered beams, fail to predict some phenomena appearing when different layers are interacting. After introducing basic definitions and key hypotheses (Section 3.1), a beam model able to properly describe the basic features of cross-sectional deformation and normal stress distributions is presented (Section 3.2). Moreover, in order to compare the proposed model (NSR model) to standard approaches, the governing equations for models retaining standard assumptions on transverse stress and strain are derived. Namely, in Sections 3.3 and 3.4, models assuming vanishing transverse normal stress (NS model) and vanishing transverse deformation ( ND model) are considered.

\subsection{Definitions and hypotheses}

\subsubsection{Geometry and materials}

Let us consider a multilayered straight-axis piezoelectric beam that is made by stacking up piezoelectric and elastic layers. We decompose the reference domain $\mathscr{B}$ as the Cartesian product of the beam axis $\mathscr{A}$ and the beam normal cross-section $\mathscr{S}$. We denote by $\mathscr{S}_{i}$ with $i \in \mathscr{I}=\{1, \ldots, n\}$, the cross-sectional part occupied by the $i$ th layer and by $n$ the total number of layers $\left(\cup_{i \in \mathscr{I}} \mathscr{S}_{i}=\mathscr{S}\right)$. Moreover, we denote by $\mathscr{I}_{\mathrm{p}}$ and $\mathscr{I}_{\mathrm{e}}\left(\mathscr{I}_{\mathrm{p}} \cup \mathscr{I}_{\mathrm{e}}=\mathscr{I}\right)$ the set of indices $i$ associated with the piezoelectric and elastic layers, respectively. The following geometric and material properties are assumed: (i) the cross-sectional part $\mathscr{S}_{i}$ is rectangular and it is characterized by the width $a_{i}$ and thickness $h_{i}$; (ii) each layer is materially homogeneous and either orthotropic or transversely isotropic with respect to an axis oriented along its thickness (in particular the 
piezoelectric layers are polarized along the thickness); (iii) the upper and lower surfaces of the piezoelectric layers are covered by a conductive layer with negligible mechanical properties, the lateral ones are bare; (iv) the electrodes of the piezoelectric layers are parallel connected one to each other, and the whole beam is electrically accessible only through two external electric terminals; for each piezoelectric layer, we define a constant $\omega_{i}= \pm 1$ defining the electric connection scheme between the electrodes of the $i$ th layer and the external terminals ( $\omega_{i}=1$ in-phase connection, $\omega_{i}=-1$ counter-phase connection).

A global reference frame $\mathscr{C}=\left\{\mathbf{o}, \mathbf{e}_{1}, \mathbf{e}_{2}, \mathbf{e}_{3}\right\}$ and $n$ local reference frames $\mathscr{C}^{(i)}=\left\{\mathbf{o}^{(i)} \equiv \mathbf{o}+\bar{z}_{i} \mathbf{e}_{3}, \mathbf{e}_{1}, \mathbf{e}_{2}, \mathbf{e}_{3}\right\}$ are fixed once for all, as sketched in Fig. 1. The coordinate representation in $\mathscr{C}$ of a generic point $\mathbf{p} \in \mathscr{B}$ is denoted by $(x, y, z)$. The local $\mathbf{e}_{3}$-coordinate in $\mathscr{C}^{(i)}$ is denoted by $z_{i}=z-\bar{z}_{i}$, where $\bar{z}_{i}$ is chosen in such a way that

$$
\int_{\mathscr{S}_{i}} z_{i} \mathrm{~d} \mathscr{S}=0 .
$$

\subsubsection{Hypotheses}

With the proposed mixed variational formulation, the reduced model generalized forces and the associated equilibrium equations are determined by hypotheses for $(\mathbf{u}, \varphi)$. On the other hand, hypotheses for (S, D) affect the associated constitutive prescription. Here, in order to introduce the minimum number of kinematical descriptors, an elementary Euler-Bernoulli-like electromechanical kinematics is adopted. At the same time, the estimate of the associated constitutive prescriptions is improved by carefully selecting the cross-sectional distribution of mechanical stress and electric displacement.

In order to derive efficient and accurate beam models of layered piezoelectric composites accounting for the two-fold electromechanical coupling, we assume the following hypotheses for the distribution of the electromechanical state fields over a beam cross-section.

(H1) Mechanical displacement. Basic equivalent-single-layer Euler-Bernoulli kinematics:

$$
\mathbf{u}(x, y, z)=\left(u(x)-z w^{\prime}(x)\right) \mathbf{e}_{1}+w(x) \mathbf{e}_{3},
$$

where $u(x)$ and $w(x)$ are the beam axis displacements along $\mathbf{e}_{1}$ and $\mathbf{e}_{3}$, respectively.

(H2) Electric potential. Layerwise linear distribution of the electric potential and parallel interconnection of the different layers

$$
\varphi(x, y, z)=\left(\frac{1}{2}+\omega_{i} \frac{z_{i}}{h_{i}}\right) V,
$$

where $V$ is the electric potential difference across the two electric terminals of the beam.

(H3) Mechanical stress. Layerwise linear distribution of plane-stress with vanishing shear term:

$$
\mathbf{T}(x, y, z)=\left(\sigma_{1, i}(x)-z_{i} \zeta_{1, i}(x)\right)\left(\mathbf{e}_{1} \otimes \mathbf{e}_{1}\right)+\left(\sigma_{2, i}(x)-z_{i} \zeta_{2, i}(x)\right)\left(\mathbf{e}_{2} \otimes \mathbf{e}_{2}\right),
$$

where, layer by layer, constant $\left(\sigma\right.$ 's) and linear ( $\left.\zeta^{\prime} s\right)$ contributions to the beam stress in the axial $\left(\mathbf{e}_{1}\right)$ and transverse $\left(\mathbf{e}_{2}\right)$ directions are defined.

(H4) Electric displacement. Layerwise constant distribution along the thickness direction:

$$
\mathbf{D}(x, y, z)=\mathrm{D}_{3, i}(x) \mathbf{e}_{3} .
$$

Particular attention is devoted to the discussion of the consequences (and the plausibility) of hypotheses about the distribution of transverse normal stress and strain $T_{22}=\mathbf{T e}_{2} \cdot \mathbf{e}_{2}$ and $S_{22}=\mathbf{S e}_{2} \cdot \mathbf{e}_{2}$. Models assuming the following three different further conditions on transverse normal stress $T_{22}$ are analyzed and compared. 
(C1) Null transverse stress resultants (NSR). The following conditions of vanishing stress resultants are imposed:

$$
\left\{\begin{array}{l}
N_{y}=\int_{\mathscr{S}} \mathbf{T}(x, y, z) \mathbf{e}_{2} \cdot \mathbf{e}_{2} \mathrm{~d} \mathscr{S}=0, \\
M_{y}=\int_{\mathscr{S}}-z \mathbf{T}(x, y, z) \mathbf{e}_{2} \cdot \mathbf{e}_{2} \mathrm{~d} \mathscr{S}=0 .
\end{array}\right.
$$

(C2) Null transverse stress (NS). The following condition of pointwise null transverse stress is enforced:

$$
T_{22}=\mathbf{T}(x, y, z) \mathbf{e}_{2} \cdot \mathbf{e}_{2}=0 .
$$

(C3) Null transverse deformation (ND). We allow the stress fields of the type specified by (H3) to vary freely. In this case the distribution of the transverse normal stress $T_{22}$ will be determined in order to retain condition of null transverse deformation implied by hypothesis (H1).

The different hypotheses on the electromechanical state fields and their influence on the properties of the associated beam models deserve some comments.

(i) Notwithstanding the elementary Euler-Bernoulli kinematics in hypothesis (H1), the beam constitutive prescriptions will include the influence of cross-sectional deformations and transversal interactions between different layers through hypotheses on transverse stress (i.e. (H3) combined with one between conditions (C1), (C2), and (C3)). Indeed, the influence of a sectional distension along the thickness is implicitly taken into account by enforcing null normal stress $T_{33}$. Furthermore, the influence of sectional extensional deformations along $\mathbf{e}_{2}$ are introduced by constraining the admissible transverse normal stress $T_{22}$.

(ii) The linear distribution of the electric potential assumed in $(\mathrm{H} 2)$ specifies the electric kinematics only in terms of the potential difference $V$. However, because of hypothesis $(\mathrm{H} 4)$, the beam constitutive equations are derived by assuming a layerwise constant electric displacement. As extensively detailed in Sze et al. (in press), the hypothesis of constant electric displacement is the best suited for fitting the three-dimensional distribution of the electric state fields without introducing additional electric degrees of freedom. In this way, as can be checked by writing down the constitutive equation for the electric potential, a through-thethickness linear contribution to the electric field (corresponding to a quadratic electric potential) is implicitly associated with flexural strains. One of the advantage of the proposed mixed variational formulation is to straightforwardly include in the model the hypothesis of layerwise constant electric displacement.

(iii) Hypotheses (H3) and (H4) introduce in the beam constitutive equations the effects of cross-sectional deformations and quadratic distribution of the electric potential. However, the electrical and mechanical shear-like effects associated with the axial variations of cross-sectional displacements and electric potential are neglected. In other words: the piezoelectric laminate is modelled as beam axis on which orthogonal cross-sections are positioned; for given axial displacements these cross-sections, being constrained to remain orthogonal to the axis, are left free to deform in their own plane so as to fit the conditions on the distribution of transverse stress; moreover, for a given potential difference $V$, the electric potential inside the piezoelectric layers is left free to vary along the thickness in order to satisfy the condition (H4) of constant electric displacement; however, since all the shear-like electrical and mechanical effects are discarded, all shear interactions between two adjacent beam cross-sections are neglected and only those exerted through axial normal stress are retained.

(iv) Conditions $(\mathrm{C} 1)$ and $(\mathrm{C} 2)$ restrict the admissible stress distributions over a section. Condition (C2) implies (C1). In the case of a homogeneous single-layer beam, given hypothesis (H3), also the inverse is true and the two conditions are equivalent. This is not the case of a multi-layered beam. In the following, we refer to the NS condition $(\mathrm{Cl})$ also as to the strong condition on transverse stress and to the NSR condition 
(C2) also as the weak condition on transverse stress. The weak condition can be interpreted as an integral version of the force balance in the transverse direction. As it will be shown in the following, in a multilayered beam transverse interactions between different layers are present and the transverse normal stress is not vanishing pointwise. In this case, the strong condition (C2) is not physically grounded, while the weakened version $(\mathrm{C} 3)$ is still legitimized by global balance laws.

In the following, the three beam models accounting for assumption (H1)-(H4) and corresponding to conditions $(\mathrm{C} 1),(\mathrm{C} 2)$ or $(\mathrm{C} 3)$ are deduced by a mixed variational formulation based on the HellingerPrange-Reissner functional (6). The additional conditions (13) and (14) on transverse normal stress are imposed on the variational problem by the Lagrange multipliers method. The following external actions are included in the description: (i) a force distribution on the beam bases having a force resul$\operatorname{tant} \mathbf{F}=\bar{N} \mathbf{e}_{1}+\bar{T} \mathbf{e}_{3}$ and a moment resultant $\mathbf{M}=-\bar{M} \mathbf{e}_{2}$; (ii) a body force per unit volume $\mathbf{b}(x, y, z)=$ $b(x, y, z) \mathbf{e}_{3}$; (iii) either a voltage $\bar{V}$ or a total charge $\bar{Q}$ imposed on the electric terminal of the set of piezoelectric layers.

\subsubsection{Beam state fields}

When hypotheses (H1) and (H3) are assumed, the three-dimensional distribution of the mechanical displacement and the electric field is given as function of the following axial fields

$$
\begin{aligned}
& \mathrm{U}=\left[\begin{array}{ll}
u & w
\end{array}\right], \\
& \mathrm{V}=[V] .
\end{aligned}
$$

Moreover, the strain tensor and the electric fields in the $i$ th layer are in the form

$$
\begin{aligned}
& \mathbf{S}(x, y, z)=\left(\varepsilon_{1, i}(x)-z_{i} \kappa_{1, i}(x)\right) \mathbf{e}_{1} \otimes \mathbf{e}_{1}, \\
& \mathbf{E}(x, y, z)=\mathrm{E}_{3, i} \mathbf{e}_{3},
\end{aligned}
$$

where

$$
\varepsilon_{1, i}(x)=u^{\prime}(x)-\bar{z}_{i} w^{\prime \prime}(x), \quad \kappa_{1, i}(x)=w^{\prime \prime}(x)
$$

and

$$
\mathrm{E}_{3, i}=-\frac{\omega_{i}}{h_{i}} V .
$$

Hence, the 3D distribution of the mechanical deformation and electric field is specified by the axial fields (here and henceforth the explicit dependence on the space variables is omitted)

$$
\mathrm{S}=\left\{\varepsilon_{1, i}, \kappa_{1, i}\right\}_{i=1}^{n}, \quad \mathrm{E}=\left\{\mathrm{E}_{3, i}\right\}_{i=1}^{n} .
$$

We say that the fields $(\mathrm{U}, \mathrm{S})$ are compatible if they are related through the compatibility relations (17) and if the displacement $\mathbf{u}$ induced by $\mathrm{U}$ through (9) verifies the geometric boundary condition $\mathbf{u}=\mathbf{u}_{0}$ on $\partial_{\mathbf{u}} \mathscr{B}$. In the same way, we say that $(\mathrm{V}, \mathrm{E})$ are compatible if they are related through the compatibility relations (18) and if the electric potential $\varphi$ induced by $\vee$ through (10) verifies the geometric boundary condition $\varphi=\varphi_{0}$ on $\partial_{\varphi} \mathscr{B}$. In what follows, we denote by $\mathscr{W}_{\mathbf{u}}$ the functional space of compatible mechanical kinematical fields $(\mathrm{U}, \mathrm{S})$ and by $\mathscr{W}_{\varphi}$ that of compatible electric kinematical fields $(\mathrm{V}, \mathrm{E})$.

By the assumed distribution for the mechanical stress and the electric displacement, the beam dynamic state is specified through the generalized stress descriptors

$$
\mathrm{T}=\left\{\sigma_{1, i}, \zeta_{1, i}, \sigma_{2, i}, \zeta_{2, i}\right\}_{i=1}^{n},
$$




$$
\mathrm{D}=\left\{\mathrm{D}_{3, i}\right\}_{i=1}^{n} .
$$

Let us define $\mathscr{W}_{\mathbf{T}}$ as the functional space of the suitably regular beam stress descriptors $\mathrm{T}$ and $\mathscr{W}_{\mathbf{D}}$ as the functional space of the beam electric displacement descriptors D. Hence, the fields in the space $\mathscr{W} \equiv \mathscr{W}_{\mathbf{u}} \times \mathscr{W}_{\varphi} \times \mathscr{W}_{\mathbf{T}} \times \mathscr{W}_{\mathbf{D}}$ characterize the electromechanical state of the reduced beam model.

The definitions above will be extensively used for the formulation of the variational problem associated with the different beam models.

\subsection{Model with null transverse stress resultants (NSR model)}

The variational problem for the beam model corresponding to hypotheses (H1)-(H4) and to the NSR condition on transverse stress is formulated by rewriting the reduced beam version of the HellingerPrange-Reissner functional (6) by taking into account hypotheses (H1)-(H4) and successively by enforcing the weak conditions (13) on transverse stresses by the Lagrange multiplier method. Associated beam balance and constitutive equations are found by looking for the stationary point of the so obtained beam functional. In particular, the Euler equations found by varying mechanical and electrical kinematical fields $(\mathrm{U}, \mathrm{V})$ furnish mechanical and electrical equilibrium equations; those found by varying mechanical and electrical dynamic fields (T, D) furnish mechanical and electrical constitutive equations. The NSR conditions are recovered by imposing the beam functional to be stationary with respect to variations of the introduced Lagrange multipliers.

\subsubsection{Variational formulation}

Let us define the following beam model free energy

$$
\mathscr{F} \text { beam }=\int_{\mathscr{S}} \mathscr{F}(\mathbf{T}, \mathbf{D}) \mathrm{d} \mathscr{S} \text {. }
$$

By making use of hypotheses (H1)-(H4) it becomes

$$
\mathscr{F}_{\text {beam }}(\mathrm{T}, \mathrm{D})=\sum_{i \in \mathscr{I}} \frac{1}{2}\left(A_{i} s_{\alpha \beta, i} \sigma_{\alpha, i} \sigma_{\beta, i}+J_{i} s_{\alpha \beta} \zeta_{\alpha, i} \zeta_{\beta, i}\right)+\sum_{i \in \mathscr{G}_{\mathrm{p}}} A_{i} g_{3 \alpha, i} \sigma_{\alpha, i} \mathrm{D}_{3, i}-\sum_{i \in \mathscr{I}_{\mathrm{p}}} \frac{1}{2} \beta_{33, i}^{\mathrm{T}} A_{i} \mathrm{D}_{3, i}^{2},
$$

where

$$
A_{i}=\int_{\mathscr{S}} \mathrm{d} \mathscr{S}=a_{i} h_{i}, \quad J_{i}=\int_{\mathscr{S}} z_{i}^{2} \mathrm{~d} \mathscr{S}=a_{i} h_{i}^{3} / 12
$$

and an implicit summation over $\alpha, \beta \in\{1,2\}$ is implied. The constitutive coefficients $s_{\alpha \beta, i}, g_{3 \alpha, i}$, and $\beta_{33, i}^{\mathrm{T}}$ are defined with the standard notation for piezoelectric materials except for dropping the superscript $D$ (null electric displacement) on $s$ and adding a subscript $i$ to distinguish the constitutive properties of different layers. Due to material symmetries $g_{32, i}=g_{31, i}, s_{12, i}=s_{21, i}, s_{11, i}=s_{22, i}$.

Hence, by substituting hypotheses (H1)-(H4), the Hellinger-Prange-Reissner functional $\Theta$ defined in Eq. (6) becomes,

$$
\begin{aligned}
\Theta_{\text {beam }}[(\mathrm{U}, \mathrm{S}),(\mathrm{V}, \mathrm{E}), \mathrm{T}, \mathrm{D}]= & \int_{\mathscr{A}} \mathscr{F}_{\text {beam }}(\mathrm{T}, \mathrm{D}) \mathrm{d} x+\sum_{i \in \mathscr{I}_{\mathrm{p}}} \int_{\mathscr{A}} A_{i} \mathrm{D}_{3, i} \mathrm{E}_{3, i} \mathrm{~d} x-\sum_{i \in \mathscr{I}} \int_{\mathscr{A}}\left(A_{i} \sigma_{1, i} \varepsilon_{1, i}+J_{i} \zeta_{1, i} \kappa_{1, i}\right) \mathrm{d} x \\
& +\int_{\mathscr{A}}\left(b_{N} u+b_{M} w^{\prime}+b_{\mathrm{T}} w\right) \mathrm{d} x+\left[\bar{N} u+\bar{M} w^{\prime}+\bar{T} w\right]_{\mathscr{A}}-\bar{Q} V
\end{aligned}
$$


where

$$
\begin{aligned}
& b_{N}=\int_{\partial_{f} \mathscr{S}} \mathbf{f}_{0} \cdot \mathbf{e}_{1} \mathrm{~d} \ell+\int_{\mathscr{S}} \mathbf{b} \cdot \mathbf{e}_{1} \mathrm{~d} \mathscr{S}, \quad \bar{N}=\int_{\mathscr{S}} \mathbf{f}_{0} \cdot \mathbf{e}_{1} \mathrm{~d} \mathscr{S}, \\
& b_{M}=-\int_{\partial_{f} \mathscr{S}} z \mathbf{f}_{0} \cdot \mathbf{e}_{1} \mathrm{~d} \ell-\int_{\mathscr{S}}\left(z \mathbf{b} \cdot \mathbf{e}_{1}\right) \mathrm{d} \mathscr{S}, \quad \bar{M}=-\int_{\mathscr{S}} z \mathbf{f}_{0} \cdot \mathbf{e}_{1} \mathrm{~d} \mathscr{S}, \\
& b_{\mathrm{T}}=\int_{\partial_{f} \mathscr{S}} \mathbf{f}_{0} \cdot \mathbf{e}_{3} \mathrm{~d} \ell+\int_{\mathscr{S}} \mathbf{b} \cdot \mathbf{e}_{3} \mathrm{~d} \mathscr{S}, \quad \bar{T}=\int_{\mathscr{S}} \mathbf{f}_{0} \cdot \mathbf{e} \mathrm{d} \mathscr{S},
\end{aligned}
$$

and $\bar{Q}$ is the total charge imposed on the two electric terminals of the multilayered beam. The reduced beam functional (25) is defined over the space $\mathscr{W} \equiv \mathscr{W}_{\mathbf{u}} \times \mathscr{W}_{\varphi} \times \mathscr{W}_{\mathbf{T}} \times \mathscr{W}_{\mathbf{D}}$.

The variational formulation is completed by imposing the weak conditions (13) on transverse stress by the Lagrange multiplier method. Hence, the following modified functional is introduced

$$
\tilde{\Theta}_{\text {beam }}=\Theta_{\text {beam }}+\tilde{\Theta}_{\text {beam }}^{(\Lambda)}
$$

with

$$
\tilde{\Theta}_{\text {beam }}^{(\Lambda)}=-\int_{\mathscr{A}} \lambda(x)\left(\int_{\mathscr{S}} \mathbf{T}(x, y, z) \mathbf{e}_{2} \cdot \mathbf{e}_{2} \mathrm{~d} \mathscr{S}\right) \mathrm{d} x-\int_{\mathscr{A}} \mu(x)\left(\int_{\mathscr{S}}-z \mathbf{T}(x, y, z) \mathbf{e}_{2} \cdot \mathbf{e}_{2} \mathrm{~d} \mathscr{S}\right) \mathrm{d} x .
$$

If the following generalized transverse strains are defined

$$
\left\{\begin{array}{l}
\varepsilon_{2, i}=\lambda(x)-\bar{z}_{i} \mu(x) \\
\kappa_{2, i}=\mu(x)
\end{array}\right.
$$

then $\tilde{\Theta}_{\text {beam }}^{(\Lambda)}$ can be rewritten as

$$
\tilde{\Theta}_{\text {beam }}^{(\Lambda)}=-\int_{\mathscr{A}} \sum_{i \in \mathscr{I}}\left(A_{i} \varepsilon_{2, i} \sigma_{2, i}+J_{i} \kappa_{2, i} \zeta_{2, i}\right) \mathrm{d} x .
$$

Finally, the constrained variational problem associated with the NSR model consists of looking for the stationary point of the modified functional $\tilde{\Theta}_{\text {beam }}$ over the extended space

$$
\widetilde{\mathscr{W}} \equiv \mathscr{W}_{\mathbf{u}} \times \mathscr{W}_{\varphi} \times \mathscr{W}_{\mathbf{T}} \times \mathscr{W}_{\mathbf{D}} \times \mathscr{W}_{\Lambda},
$$

where $\mathscr{W}_{\Lambda}$ is the space of admissible Lagrange multipliers $\Lambda=\{\lambda, \mu\}$.

The transverse strains $\left(\varepsilon_{2, i}, \kappa_{2, i}\right)$ are the analog of $\left(\varepsilon_{1, i}, \kappa_{1, i}\right)$ in the width direction and they can be regarded as the constant and linear part of the deformation along $\left(\mathbf{e}_{2} \otimes \mathbf{e}_{2}\right)$. Similarly, the Lagrange multipliers $(\lambda, \mu)$ are the analog of $\left(u^{\prime}, w^{\prime \prime}\right)$ in the transverse direction. They are associated with the extensional $(\lambda)$ and flexural $(\mu)$ sectional deformation in the transverse direction, exactly as $\left(u^{\prime}, w^{\prime \prime}\right)$ are in the axial direction.

\subsubsection{Balance equations}

The balance equations and the natural boundary conditions are derived by imposing that the first variation of the functional (27) in $\mathscr{W}_{\mathbf{u}} \times \mathscr{W}_{\varphi}$ is null. Hence, the following balance equations are found:

$$
\left\{\begin{array}{l}
b_{N}+N_{x}^{\prime}=0, \\
b_{M}^{\prime}-b_{\mathrm{T}}+M_{x}^{\prime \prime}=0, \\
\int_{\mathscr{A}} q \mathrm{~d} x-\bar{Q}=0,
\end{array}\right.
$$


where the following force resultants have been defined

$$
\begin{aligned}
& N_{x}=\sum_{i \in \mathscr{I}} \int_{S_{i}} \mathbf{T} \cdot\left(\mathbf{e}_{1} \otimes \mathbf{e}_{1}\right) \mathrm{d} S=\sum_{i \in \mathscr{I}} A_{i} \sigma_{1, i} \\
& M_{x}=\sum_{i \in \mathscr{I}} \int_{S_{i}}-z \mathbf{T} \cdot\left(\mathbf{e}_{1} \otimes \mathbf{e}_{1}\right) \mathrm{d} S=-\sum_{i \in \mathscr{I}} \bar{z}_{i} A_{i} \sigma_{1, i}+\sum_{i \in \mathscr{I}} J_{i} \zeta_{1, i}, \\
& q=-\sum_{i \in \mathscr{I}_{\mathrm{p}}} \omega_{i} a_{i} \mathrm{D}_{3, i} .
\end{aligned}
$$

The natural boundary conditions are derived by imposing that the following conditions are satisfied for all the admissible variations $\delta u$ and $\delta w$ of $u$ and $w$ in $\mathscr{W}_{\mathbf{u}}$

$$
\left\{\begin{array}{l}
{\left[\left(N_{x}-\bar{N}\right) \delta u\right]_{\partial \mathscr{A}}=0} \\
{\left[\left(M_{x}-\bar{M}\right) \delta w^{\prime}+\left(\bar{T}+M_{x}^{\prime}+b_{M}\right) \delta w\right]_{\partial \mathscr{A}}=0 .}
\end{array}\right.
$$

As expected because of the assumed kinematics, the balance equations above correspond to those of a standard Euler-Bernoulli beam model.

\subsubsection{Constitutive equations}

Local constitutive equations. Local constitutive equation valid layer by layer are found by imposing that the first variation of the functional (27) with respect to (T,D) is null. They are given by

$$
\text { piezoelectric layers }\left\{\begin{array} { l } 
{ s _ { \alpha \in \mathscr { J } _ { \mathrm { p } } } ^ { \mathrm { D } } \sigma _ { \beta , i } + g _ { 3 \alpha } \mathrm { D } _ { 3 , i } = \varepsilon _ { \alpha , i } , } \\
{ s _ { \alpha \beta } ^ { \mathrm { D } } \zeta _ { \beta , i } = \kappa _ { \alpha , i } , } \\
{ - g _ { 3 \alpha } \sigma _ { \alpha , i } + \beta _ { 3 3 } ^ { \mathrm { T } } \mathrm { D } _ { 3 , i } = \mathrm { E } _ { 3 , i } ^ { ( l ) } , }
\end{array} \quad \text { elastic layers } \left\{\begin{array}{l}
s_{\alpha \beta} \sigma_{\beta, i}=\varepsilon_{\alpha, i}, \\
s_{\alpha \beta} \zeta_{\beta, i}=\kappa_{\alpha, i} .
\end{array}\right.\right.
$$

The constitutive equations above can be inverted and rewritten in the form

$$
\text { piezoelectric layers }\left\{\begin{array} { l } 
{ \sigma _ { \alpha , i } = \tilde { c } _ { \alpha \beta } ^ { \mathrm { E } } \varepsilon _ { \beta , i } - \tilde { e } _ { 3 \beta } \mathrm { E } _ { 3 , i } , } \\
{ \zeta _ { \alpha , i } = \tilde { c } _ { \alpha \beta } ^ { \mathrm { D } } \kappa _ { \beta , i } , } \\
{ \mathrm { D } _ { 3 , i } = \tilde { e } _ { 3 \beta } \varepsilon _ { \beta , i } + \tilde { \epsilon } _ { 3 3 } ^ { \mathrm { S } } \mathrm { E } _ { 3 , i } }
\end{array} \quad \text { elastic layers } \left\{\begin{array}{l}
\sigma_{\alpha, i}=\tilde{\mathcal{T}}_{\alpha \beta} \varepsilon_{\beta, i}, \\
\zeta_{\beta, i}=\tilde{c}_{\alpha \beta} \kappa_{\beta, i} .
\end{array}\right.\right.
$$

Explicit expressions for the new constitutive coefficients are given in Appendix A.1. As it can be easily checked, their expressions correspond to those of the constitutive coefficient of piezoelectric and elastic materials in which the normal stress along $\mathbf{e}_{3}$ (namely, $T_{33}$ ) is imposed to be null and the electric displacement constant along the thickness. This is consequence of assumption (H3) where $T_{33}=\mathbf{T e}_{3} \cdot \mathbf{e}_{3}=0$ and assumption (H4) where $\partial \mathbf{D} / \partial z=0$.

Conditions on transverse stresses. The NSR conditions on transverse stresses given by Eqs. (13) are retrieved by imposing that the first variation of the beam functional (27) with respect to the Lagrange multipliers $(\lambda, \mu)$ is null. By introducing the following force resultants

$$
N_{y}=\sum_{i \in \mathscr{I}} A_{i} \sigma_{2, i}, \quad M_{y}=\sum_{i \in \mathscr{I}} J_{i} \zeta_{2, i}-\bar{z}_{i} A_{i} \sigma_{2, i},
$$

they appear in the form

$$
\left\{\begin{array}{l}
N_{y}=0 \\
M_{y}=0 .
\end{array}\right.
$$


Global constitutive equations. Let us collect the force resultants in the axial and transverse direction in the following vectors:

$$
\mathrm{R}_{x}=\left[\begin{array}{l}
N_{x} \\
M_{x}
\end{array}\right], \quad \mathrm{R}_{y}=\left[\begin{array}{l}
N_{y} \\
M_{y}
\end{array}\right] .
$$

Moreover, let us introduce the vectors collecting the axial fields representing beam deformations in the two directions as follows

$$
\mathrm{d}_{x}=\left[\begin{array}{c}
u^{\prime} \\
w^{\prime \prime}
\end{array}\right], \quad \mathrm{d}_{y}=\left[\begin{array}{l}
\lambda \\
\mu
\end{array}\right]=\Lambda,
$$

where the Lagrange multipliers have been explicitly associated with transversal extension $(\lambda)$ and flexion $(\mu)$ of the beam cross-section. Hence, by substituting the local constitutive equations (36) and the kinematic compatibility equations (16), (17), (18) in the definitions of the force resultants (33)-(37), the global beam constitutive equations in the following form are found:

$$
\left[\begin{array}{c}
\mathrm{R}_{x} \\
\mathrm{R}_{y} \\
q
\end{array}\right]=\left[\begin{array}{ccc}
K_{x x} & K_{x y} & -e_{\mathrm{d} V}^{t} \\
K_{x y} & K_{x x} & -e_{\mathrm{d} V}^{t} \\
e_{\mathrm{d} V} & e_{\mathrm{d} V} & \epsilon_{q V}
\end{array}\right]\left[\begin{array}{c}
\mathrm{d}_{x} \\
\mathrm{~d}_{y} \\
V
\end{array}\right]
$$

where

$$
K_{x x}=\left[\begin{array}{cc}
k_{N u} & -k_{N w} \\
-k_{N w} & k_{M w}
\end{array}\right], \quad K_{x y}=\left[\begin{array}{cc}
k_{N \lambda} & -k_{N \mu} \\
-k_{N \mu} & k_{M \mu}
\end{array}\right], \quad e_{\mathrm{d} V}=\left[\begin{array}{ll}
-e_{N V} & e_{M V}
\end{array}\right] .
$$

Explicit expressions for the constitutive constants appearing above in terms of three-dimensional material and constitutive properties are reported in Appendix A.2.

The conditions on transverse stresses (38) impose that

$$
\mathrm{R}_{y}=K_{x y} \mathrm{~d}_{x}+K_{x x} \mathrm{~d}_{y}-e_{\mathrm{d} V}^{t} V=0 .
$$

Hence, the following expressions for the transverse deformations are found

$$
\mathrm{d}_{y}=-K_{x x}^{-1} K_{x y} \mathrm{~d}_{x}+K_{x x}^{-1} e_{\mathrm{d} V}^{t} V .
$$

Finally, the following constrained global constitutive equations are obtained by substituting the expressions (44) for the transverse deformations in the constitutive equations (41)

$$
\left[\begin{array}{c}
\mathrm{R}_{x} \\
q
\end{array}\right]=\left[\begin{array}{cc}
K_{x x}^{(\mathrm{NSR})} & -\left(e_{\mathrm{d} V}^{(\mathrm{NSR})}\right)^{t} \\
e_{\mathrm{d} V}^{(\mathrm{NSR})} & \epsilon_{\mathrm{d} V}^{t(\mathrm{NSR})}
\end{array}\right]\left[\begin{array}{c}
\mathrm{d}_{x} \\
V
\end{array}\right],
$$

where

$$
\left\{\begin{array}{c}
K_{x x}^{(\mathrm{NSR})}=K_{x x}-K_{x y} K_{x x}^{-1} K_{x y}, \\
e_{\mathrm{d} V}^{(\mathrm{NSR})}=e_{\mathrm{d} V}-e_{\mathrm{d} V} K_{x x}^{-1} K_{x y}, \\
\epsilon_{q V}^{(\mathrm{NSR})}=\epsilon_{q V}+e_{\mathrm{d} V} K_{x x}^{-1} e_{\mathrm{d} V}^{t} .
\end{array}\right.
$$

If the different layers are symmetrically disposed with respect to a given plane or if $c_{12}^{\mathrm{E}} / c_{11}^{\mathrm{E}}=c_{12} / c_{11}$, it is possible to choose the position of the $\mathbf{e}_{2}$ axis is such a way that

$$
k_{N w}=k_{N \mu}=0,
$$

and the matrices $K_{x x}$ and $K_{x y}$ are diagonal. Therefore, the constrained beam constitutive equations (45) are written as 


$$
\left[\begin{array}{c}
N_{x} \\
M_{x} \\
q
\end{array}\right]=\left[\begin{array}{ccc}
k_{N u}^{(\mathrm{NSR})} & 0 & -e_{N V}^{(\mathrm{NSR})} \\
0 & k_{M w}^{(\mathrm{NSR})} & e_{M V}^{(\mathrm{NSR})} \\
e_{N V}^{(\mathrm{NSR})} & -e_{M V}^{(\mathrm{NSR})} & \epsilon_{q V}^{\mathrm{NSR})}
\end{array}\right]\left[\begin{array}{c}
u^{\prime} \\
w^{\prime \prime} \\
V
\end{array}\right]
$$

where the constitutive coefficients are given by the following simple expressions:

$$
\begin{aligned}
& k_{N u}^{(\mathrm{NSR})}=k_{N u}\left(1-k_{N \lambda}^{2} / k_{N u}^{2}\right), \\
& k_{M w}^{(\mathrm{NSR})}=k_{M w}\left(1-k_{M \mu}^{2} / k_{M w}^{2}\right), \\
& e_{N V}^{(\mathrm{NSR})}=e_{N V}\left(1-k_{N \lambda} / k_{N u}\right), \\
& e_{M V}^{(\mathrm{NSR})}=e_{M V}\left(1-k_{M \mu} / k_{M w}\right), \\
& \epsilon_{q V}^{(\mathrm{NSR})}=\epsilon_{q V}\left(1+e_{N V}^{2} /\left(k_{N u} \epsilon_{q V}\right)+e_{M V}^{2} /\left(k_{M w} \epsilon_{q V}\right)\right) .
\end{aligned}
$$

Moreover, the relations (44) expressing the cross-sectional transverse deformations in terms of axial ones and applied electric potential are written explicitly as

$$
\mathrm{d}_{y}=\left[\begin{array}{l}
\lambda \\
\mu
\end{array}\right]=\left[\begin{array}{ccc}
-k_{N \lambda} / k_{N u} & 0 & -e_{N V} / k_{N u} \\
0 & -k_{M \mu} / k_{M w} & e_{M w} / k_{M w}
\end{array}\right]\left[\begin{array}{c}
u^{\prime} \\
w^{\prime \prime} \\
V
\end{array}\right] .
$$

The expressions above for cross-sectional transverse extension and bending show how, in order to enforce the NSR conditions (38), the constitutive equations of the NSR model (45) account for cross-sectional deformations. This feature is provided by the mixed variational formulation, where the beam constitutive behavior is prescribed through the hypotheses on generalized stress descriptors. In this way, despite the assumed elementary Euler-Bernoulli kinematics, beam cross-sectional deformations are implicitly allowed for by suitably restricting admissible stress states.

\subsection{Model with pointwise null transverse stress (NS model)}

\subsubsection{Variational formulation}

The strong conditions (14) on transverse stress naturally restrict the space of admissible stress to the subspace $\widehat{\mathscr{W}}_{\mathrm{T}}$ of $\mathscr{W}_{\mathrm{T}}$ made of the generalized stress $\mathrm{T}$ characterized by $\sigma_{2, i}=\zeta_{2, i}=0$. Hence, the variational problem in this case could be stated as finding the stationary point of (25) over $\mathscr{W}_{\mathbf{u}} \times \mathscr{W}_{\varphi} \times \widehat{\mathscr{W}}_{\mathbf{T}} \times \mathscr{W}_{\mathbf{D}}$. However, the NS conditions (14) are imposed here by the Lagrange multiplier method, in order to emphasize analogies and differences with respect to the model assuming the NSR conditions (13). Hence, the following modified functional is introduced

$$
\widehat{\Theta}_{\text {beam }}=\Theta_{\text {beam }}+\widehat{\Theta}_{\text {beam }}^{(1)},
$$

where, in view of hypothesis (H3), the additional term enforcing the NS condition (14) is given by

$$
\widehat{\Theta}_{\text {beam }}^{(\Lambda)}=-\int_{\mathscr{A}} \sum_{i \in \mathscr{I}}\left(\lambda_{i}(x) \sigma_{2, i}(x)+\mu_{i}(x) \zeta_{2, i}(x)\right) .
$$


By introducing the functional space of admissible Lagrange multipliers $\widehat{\mathscr{W}}_{\Lambda}=\left\{\lambda_{1}, \mu_{1}, \ldots, \lambda_{n}, \mu_{n}\right\}$, the variational problem associated with the NS model is finally formulated as finding the stationary set of the functional $\widehat{\Theta}_{\text {beam }}$ over

$$
\widehat{\mathscr{W}} \equiv \mathscr{W}_{\mathbf{u}} \times \mathscr{W}_{\varphi} \times \mathscr{W}_{\mathbf{T}} \times \mathscr{W}_{\mathbf{D}} \times \widehat{\mathscr{W}}_{\Lambda} .
$$

\subsubsection{Balance equations}

The equilibrium equations and the natural boundary conditions are found by taking the variation of the functional (51) in $\mathscr{W}_{\mathbf{u}} \times \mathscr{W}_{\varphi}$. As it can be easily checked, they are the same of those found for the model assuming the weak conditions on transverse stress (Eqs. (32) and (34)).

\subsubsection{Constitutive equations}

Local constitutive equations. By taking the variation of the functional (51) in $\mathscr{W}_{\mathbf{D}} \times \mathscr{W}_{\mathrm{T}}$, local constitutive equations in the form (35) and (36) are found also in this case; however the following different definitions for transverse deformations in terms of Lagrange multipliers holds:

$$
\left\{\begin{array}{l}
\varepsilon_{2, i}=\lambda_{i}, \\
\kappa_{2, i}=\mu_{i}
\end{array}\right.
$$

In this case, two Lagrange multipliers for each layer are defined. They can be interpreted as transverse cross-sectional extension and bending of each layer.

Condition on transverse stresses. The constraints on transverse stresses (14) are retrieved by imposing that the variations of the functional (51) with respect to $\left\{\lambda_{i}, \mu_{i}\right\}_{1=1}^{n}$ are null. They are

$$
\left\{\begin{array}{l}
\sigma_{2, i}=0 \\
\zeta_{2, i}=0
\end{array}\right.
$$

These conditions, being local, can be imposed directly on the local constitutive equation (35) and solved for the Lagrange multipliers $\left\{\lambda_{i}, \mu_{i}\right\}_{1=1}^{n}$. In this way, the following constrained local constitutive equations are found

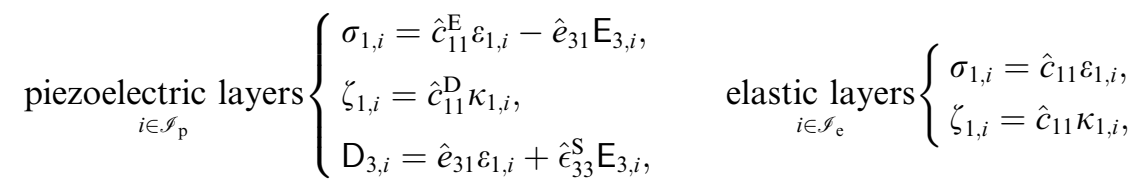

where the explicit expressions of the constitutive coefficients in terms of the three-dimensional material and geometric properties are given in Appendix A.1. As it can be easily checked, the constitutive constants with a superimposed hat correspond to those for piezoelectric and elastic materials under a uniaxial stress state in the $\mathbf{e}_{1}$-direction (i.e. for $T_{22}=T_{33}=0$ ). Moreover, the following expressions for the transverse sectional deformations of each layer are found:

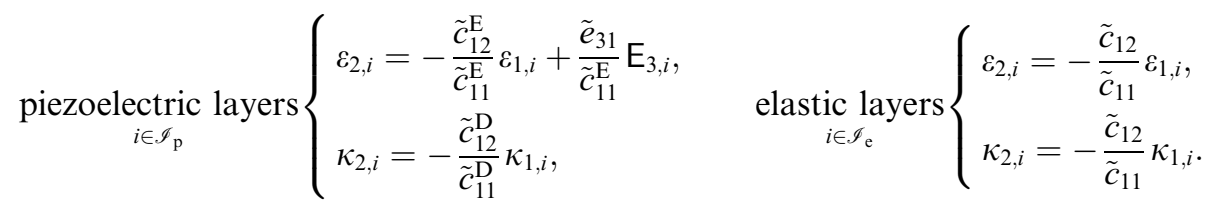


The main difference with respect to the model in which weak conditions on transverse stress are enforced, consists of neglecting the interactions between different layers in the transverse direction. Indeed, in this case, each layer is left free to extend and bend in the transversal direction independently of the other ones.

Global constitutive equations. By substituting the local constitutive equations (56) in the stress resultant definitions, global constrained constitutive equations in the following form are found

$$
\left[\begin{array}{c}
N_{x} \\
M_{x} \\
q
\end{array}\right]=\left[\begin{array}{ccc}
k_{N u}^{(\mathrm{NS})} & k_{N w}^{(\mathrm{NS})} & -e_{N V}^{(\mathrm{NS})} \\
k_{N w}^{(\mathrm{NS})} & k_{M w}^{(\mathrm{NS})} & e_{M V}^{(\mathrm{NS})} \\
e_{N V}^{\mathrm{NS})} & -e_{M V}^{(\mathrm{NS})} & \epsilon_{q V}^{(\mathrm{NS})}
\end{array}\right]\left[\begin{array}{c}
u^{\prime} \\
w^{\prime \prime} \\
V
\end{array}\right] .
$$

Explicit expressions for the constitutive coefficients above are given in Appendix A.2.

\subsection{Model with null transverse strain (ND model)}

\subsubsection{Variational formulation}

The condition of null transverse deformation is satisfied when no further hypotheses of transverse stress distribution are imposed. The corresponding variational formulation of the problem consists of looking for the stationary set of the Hellinger-Prange-Reissner functional (25) over the space $\mathscr{W}$.

The equilibrium equations, the natural boundary conditions, and the constitutive relations are found by imposing the variations of (25) in $\mathscr{W}_{\mathbf{u}} \times \mathscr{W}_{\varphi}$ and $\mathscr{W}_{\mathbf{T}} \times \mathscr{W}_{\mathrm{D}}$ equal to zero. The only difference with respect to the models assuming further conditions on transverse normal stress is that the transverse deformations $\left(\varepsilon_{2, i}, \kappa_{2, i}\right)$ appearing in the constitutive equations in the form (35) are null in each layer. Hence, the corresponding global constitutive relations are given directly by (41) for $\mathrm{d}_{y}=0$. They are in the form

$$
\left[\begin{array}{c}
N_{x} \\
M_{x} \\
q
\end{array}\right]=\left[\begin{array}{ccc}
k_{N u}^{(\mathrm{ND})} & -k_{N w}^{(\mathrm{ND})} & -e_{N V}^{(\mathrm{ND})} \\
-k_{N w}^{(\mathrm{ND})} & k_{M w}^{(\mathrm{ND})} & e_{M V}^{(\mathrm{ND})} \\
e_{N V}^{(\mathrm{ND})} & -e_{M V}^{(\mathrm{ND})} & \epsilon_{q V}^{(\mathrm{ND})}
\end{array}\right]\left[\begin{array}{c}
u^{\prime} \\
w^{\prime \prime} \\
V
\end{array}\right]
$$

with the constitutive constants given in Appendix A.2 (Eqs. (A.11)).

\section{Results and comments}

In this section, we discuss the main differences between the proposed NSR model and the standard NS and ND models by focusing on the following points:

(1) the comparison of the estimates of the beam mechanical, electrical, and coupling constitutive coefficients as functions of the thickness ratio between the piezoelectric and elastic layers;

(2) the comparison of the through-the-thickness distribution of the 3D state fields associated with a given deformation state of the beam model (which is specified by assigning $\left(u^{\prime}, w^{\prime \prime}, V\right)$ ).

To this end, two particular configurations of piezoelectric laminated beams are considered:

(i) a sandwich three-layered beam composed of a central elastic layer on which two identical piezoelectric layers are symmetrically bonded (see Fig. 2); in particular, both of the configurations with in-phase and counter-phase electric connections between the piezoelectric layers are examined (extensional-electric coupling and flexural-electric coupling, respectively).

(ii) a two-layered beam composed of a piezoelectric and an elastic layer (see Fig. 3). 


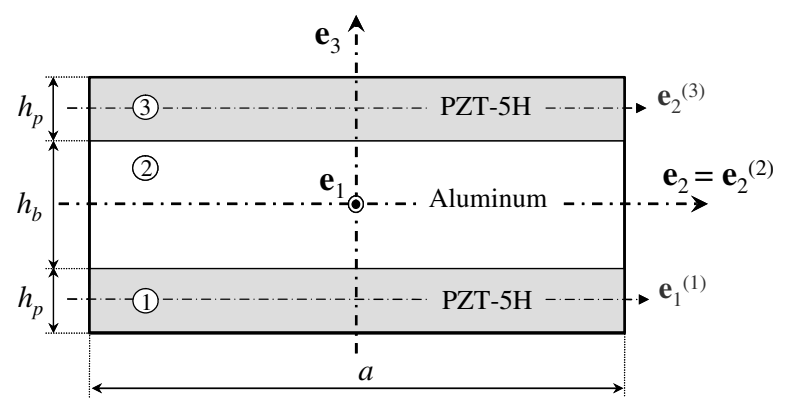

Fig. 2. Three-layered sandwich piezoelectric beam: cross-section.

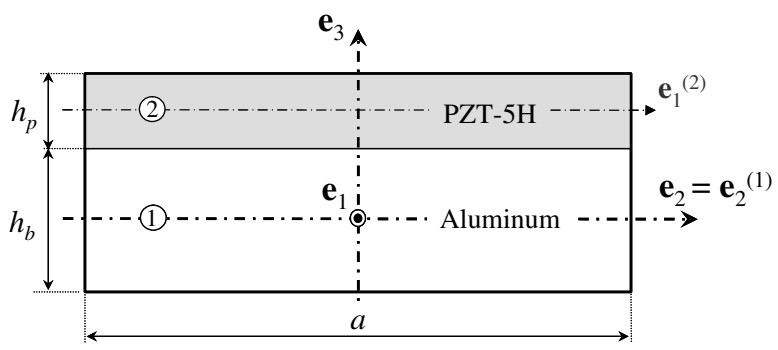

Fig. 3. Two-layered piezoelectric beam: cross-section.

In the following, we assume that the different layers all have the same width $a$. We denote by $h_{\mathrm{p}}$ the thickness of the piezoelectric layers, by $h_{\mathrm{b}}$ the thickness of the elastic layers, and we introduce the thickness ratio

$$
\eta=\frac{h_{\mathrm{b}}}{h_{\mathrm{p}}} .
$$

Elastic layers made of aluminum, and piezoelectric layers made of the piezoelectric ceramic PZT-5H are considered. The corresponding numerical values of the relevant material properties are reported in Table 1. The following dimensionless parameters are introduced:

$$
\begin{aligned}
& \tilde{\beta}_{11}=\frac{\tilde{c}_{11}}{\tilde{c}_{11}^{\mathrm{E}}}, \quad \tilde{\beta}_{12}=\frac{\tilde{c}_{12}}{\tilde{c}_{12}^{\mathrm{E}}}, \quad \hat{\beta}_{11}=\frac{\hat{c}_{11}}{\hat{c}_{11}^{\mathrm{E}}}, \\
& \tilde{\gamma}_{11}=\sqrt{\frac{\tilde{e}_{31}^{2}}{\tilde{\tilde{\varepsilon}}_{33}^{\mathrm{S}} \tilde{c}_{11}^{\mathrm{E}}}}, \quad \tilde{\gamma}_{12}=\sqrt{\frac{\tilde{e}_{31}^{2}}{\tilde{\tilde{\varepsilon}}_{33}^{\mathrm{S}} \tilde{c}_{12}^{\mathrm{E}}}}, \quad \hat{\gamma}_{11}=\sqrt{\frac{\hat{e}_{31}^{2}}{\hat{\hat{\varepsilon}}_{33}^{\mathrm{S}} \hat{c}_{11}^{\mathrm{E}}}}, \quad \tilde{v}=\frac{\tilde{c}_{12}^{\mathrm{E}}}{\tilde{c}_{11}^{\mathrm{E}}} .
\end{aligned}
$$

They represent the stiffness ratios between the elastic and piezoelectric materials $\left(\tilde{\beta}_{11}, \tilde{\beta}_{12}, \hat{\beta}_{11}\right)$, the dimensionless coupling parameters $\left(\tilde{\gamma}_{11}, \tilde{\gamma}_{12}\right)$, and the piezoelectric Poisson coefficient $(\tilde{v})$.

Table 1

Numerical values of the relevant piezoelectric and elastic constitutive coefficients for PZT 5-H and aluminum

\begin{tabular}{lllll}
\hline & $s_{11}\left(\mathrm{~m}^{2} \mathrm{~N}^{-1}\right)$ & $s_{12}\left(\mathrm{~m}^{2} \mathrm{~N}^{-1}\right)$ & $d_{31}\left(\mathrm{~m} \mathrm{~V}^{-1}\right)$ & $\beta_{33}^{\mathrm{T}}\left(\mathrm{F}^{-1} \mathrm{~m}\right)$ \\
\hline Elastic layer (aluminum) & $14.5 \times 10^{-12}$ & $-4.78 \times 10^{-12}$ & - & - \\
Piezoelectric layer (PZT-5H) & $16.1 \times 10^{-12}$ & $-4.57 \times 10^{-12}$ & $-320 \times 10^{-12}$ & $2.98 \times 10^{7}$ \\
\hline
\end{tabular}




\subsection{Constitutive coefficients}

We begin by comparing the beam constitutive coefficients relating the generalized beam stress $\left(N_{x}, M_{x}, q\right)$ to the beam generalized deformations $\left(u^{\prime}, w^{\prime \prime}, V\right)$ as obtained by the ND, NS and NSR models. In particular, for specific cross-sectional configurations (the in-phase and counter-phase connected sandwich beams and the two layered beam), the influence of the different hypotheses on transverse stress on the beam constitutive behavior as a function of the thickness ratio $\eta$ is discussed.

\subsubsection{Three-layered sandwich beam}

In this case (see Fig. 2), the layer configuration is symmetric, beam extensional and flexural modes are mechanically uncoupled $\left(k_{N w}^{(\mathrm{ND})}=k_{N \mu}^{(\mathrm{ND})}=0\right)$, and the beam constitutive equations can be evaluated from reduced expressions of the type (49). For the ND model, the following constitutive parameters are found (see expressions in Appendix A.2):

$$
\begin{aligned}
k_{N u}^{(\mathrm{ND})} & =\tilde{c}_{11}^{\mathrm{E}} a h_{\mathrm{p}} \omega_{\eta}\left(\tilde{\beta}_{11}\right), \\
k_{M w}^{(\mathrm{ND})} & =\frac{\tilde{c}_{11}^{\mathrm{E}} a h_{\mathrm{p}}^{3}}{12} \chi_{\eta}\left(\tilde{\beta}_{11}, \tilde{\gamma}_{11}\right), \\
e_{N V}^{(\mathrm{ND})} & =\left(\omega_{1}+\omega_{2}\right) a \tilde{e}_{31}, \\
e_{M V}^{(\mathrm{ND})} & =\left(\omega_{1}-\omega_{2}\right) a \tilde{e}_{31} h_{\mathrm{p}}(1+\eta) / 2, \\
\epsilon_{q V}^{(\mathrm{ND})} & =\frac{2 a}{h_{\mathrm{p}}} \tilde{\epsilon}_{33}^{\mathrm{S}},
\end{aligned}
$$

where the following functions giving the dependence on the thickness ratio $\eta$ are defined:

$$
\omega_{\eta}(\beta)=2+\eta \beta, \quad \chi_{\eta}(\beta, \gamma)=2\left(1+\gamma^{2}\right)+6(1+\eta)^{2}+\eta^{3} \beta
$$

For in-phase connected piezoelectric layers $\left(\omega_{1}=\omega_{2}=1\right)$, the potential difference between the electric terminals of the piezoelectric transducers is coupled only to the beam extensional mode. On the other hand, for counter-phase connected piezoelectric layers $\left(\omega_{1}=-\omega_{2}=1\right)$, the piezoelectric coupling involves only the beam flexural mode.

The NSR and NS models furnish different estimates of the electromechanical constitutive properties of the layered beam. We detail in Tables 2 and 3 the corresponding expressions for the mechanical extensional and flexural stiffnesses $\left(k_{N u}\right.$ and $\left.k_{M w}\right)$, coupling coefficients $\left(e_{N V}, e_{M V}\right)$, and capacitance per unit length $\epsilon_{q V}$. These quantities are reported as ratio to those of the ND model. The cases of in-phase and counter-phase electrical connections are reported separately. By assuming the numerical values of the constitutive

Table 2

Constitutive coefficients of a sandwich beam with in-phase parallel-connected piezoelectric layers

\begin{tabular}{lcc}
\hline$k_{N u} / k_{N u}^{(\mathrm{ND})}$ & NS model & NSR model \\
$e_{N V} / e_{N V}^{(\mathrm{ND})}$ & $\left(1-\tilde{v}^{2}\right) \frac{\varpi_{\eta}\left(\hat{\beta}_{11}\right)}{\varpi_{\eta}\left(\tilde{\beta}_{11}\right)}$ & $1-\tilde{v}^{2} \frac{\varpi_{\eta}^{2}\left(\tilde{\beta}_{12}\right)}{\varpi_{\eta}^{2}\left(\tilde{\beta}_{11}\right)}$ \\
$\epsilon_{q v} / \epsilon_{q v}^{(\mathrm{ND})}$ & $1-\tilde{v}$ & $1-\tilde{v} \frac{\varpi_{\eta}\left(\tilde{\beta}_{12}\right)}{\varpi_{\eta}\left(\tilde{\beta}_{11}\right)}$ \\
\hline
\end{tabular}

Ratios between the constitutive coefficients of the NS and NSR models and those of the ND model are reported. 
Table 3

Constitutive coefficients of a sandwich beam with counter-phase parallel-connected piezoelectric layers

\begin{tabular}{lcc}
\hline$k_{M w} / k_{M w}^{(\mathrm{ND})}$ & NS model & NSR model \\
$e_{M V} / e_{M V}^{(\mathrm{ND})}$ & $\left(1-\tilde{v}^{2}\right) \frac{\chi_{\eta}\left(\hat{\beta}_{11}, \hat{\gamma}_{11}\right)}{\chi_{\eta}\left(\tilde{\beta}_{11}, \tilde{\gamma}_{11}\right)}$ & $1-\tilde{v}^{2} \frac{\chi_{\eta}^{2}\left(\tilde{\beta}_{12}, \tilde{\gamma}_{12}\right)}{\chi_{\eta}^{2}\left(\tilde{\beta}_{11}, \tilde{\gamma}_{11}\right)}$ \\
$\epsilon_{q v} / \epsilon_{q v}^{\mathrm{ND})}$ & $1-\tilde{v}$ & $1-\tilde{v}_{\eta}\left(\tilde{\beta}_{12}, \tilde{\gamma}_{12}\right)$ \\
$\chi_{\eta}\left(\tilde{\beta}_{11}, \tilde{\gamma}_{11}\right)$ \\
\hline
\end{tabular}

Ratios between the constitutive coefficients of the NS and NSR models and those of the ND model are reported.
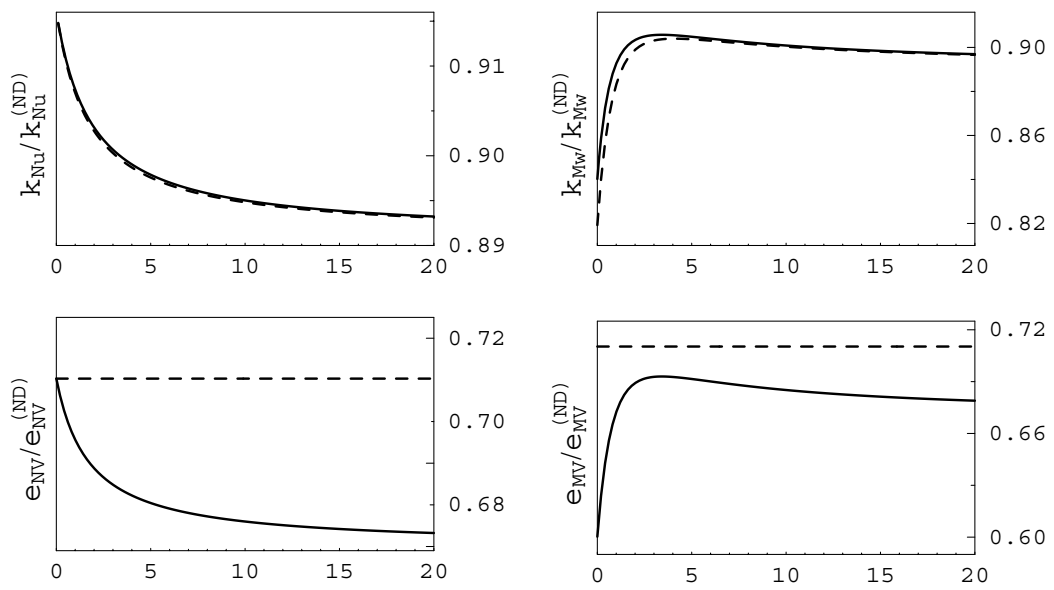

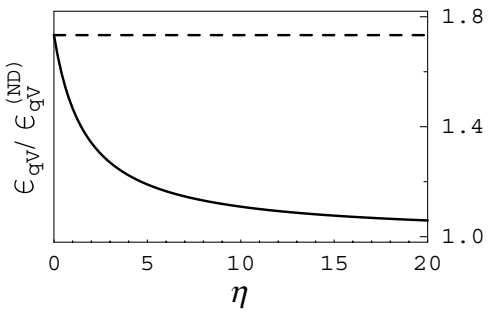

(a)

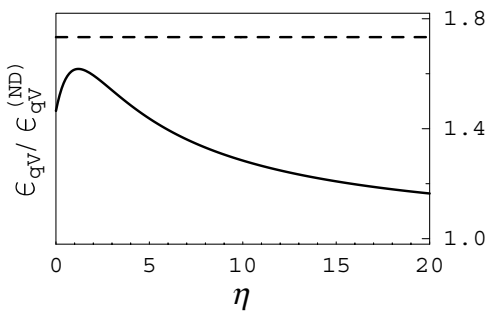

(b)

Fig. 4. Beam constitutive coefficients for the in-phase (a) and counter-phase (b) connected three-layered piezoelectric beam as a function of the thickness ratio $\eta=h_{\mathrm{b}} / h_{\mathrm{p}}$. The continuous lines refer to the NSR model, the dashed lines to the NS model. The constitutive coefficients are reported as ratios with respect to those estimated by the ND model.

coefficients in Table 1, the same quantities are plotted in Fig. 4 as a function of the thickness ratio $\eta$ for a fixed thickness of the piezoelectric layer.

From expressions (62), Tables 2 and 3, and Fig. 4, we draw the following conclusions regarding the constitutive coefficients estimated by the different models.

Electric capacitance per unit length $\left(\varepsilon_{q V}\right)$. Both for the in-phase and counter-phase electric connection between the two piezoelectric layers, the predicted value of $\varepsilon_{q V}$ strongly depends on the assumed model. In particular, once the thickness $h_{\mathrm{p}}$ of the piezoelectric layers is fixed, $\varepsilon_{q V}$ is independent of the thickness ratio $\eta$ between the piezoelectric and elastic layers in the ND and NS models. In the ND model, $\varepsilon_{q V}$ is given by the 
electric capacitance for null transverse strain, and in the NS model by that for null transverse stress; the ratio between these two values depends only on the coupling coefficient $\tilde{\gamma}_{11}$ (see Table 2 ). On the other hand, in the NSR model, the electric capacitance per unit length varies with $\eta$ : for the in-phase electric connection it is equal to the electric capacitance for null transverse strain for thick elastic substrates $(\eta \rightarrow \infty)$; it increases with $\eta$ going towards the capacitance for null transverse stress for very thin elastic substrates $(\eta \rightarrow 0)$. This behavior has a physical justification: for $\eta \rightarrow \infty$ the very thick elastic substrate behaves as a rigid element and the piezoelectric layers bonded on it cannot deform in the transverse direction; for $\eta \rightarrow 0$, the elastic layer becomes negligible and the two in-phase connected piezoelectric layers can freely deform in the transverse direction. For $\eta \rightarrow \infty$ the same considerations also hold for the counter-phase electric connection, while a different phenomenon must be considered for $\eta \rightarrow 0$. In this case, when the thickness of the elastic layer is negligible, the two counter-phase connected piezoelectric layers are in the so-called bimorph configuration: when an electric potential is applied, one tends to extend, the other to shrink. As a consequence of the bonding condition, transverse interactions between the two layers arise and transverse stresses are different from zero also for $\eta \rightarrow 0$. For this reason, when $\eta \rightarrow 0$ the electric capacitance per unit length goes toward a value different from that of the NS model.

Mechanical stiffness $\left(k_{N u}, k_{M w}\right)$. Both for in-phase and counter-phase electric connections, the mechanical stiffnesses estimated by the NS and NSR are remarkably different from that given by the ND model. As evident from relations in Tables 2 and 3, their ratio mainly depends on the value of the Poisson ratio $v$. This is a well known effect: while in the NS and NSR models the beam cross section is left free to deform in its plane, in the ND model it is constrained to be rigid. As a consequence, the ND model overestimates the extensional and flexural stiffnesses when the beam's lateral boundary is stress-free. On the contrary, for the assumed material properties of the different layers, the estimates provided by the NSR and NS models are similar, although the NS model neglects any interaction between different layers in the transverse direction (each layer is left free to deform independently from the other ones). This may be the reason why the NS condition is usually accepted for modelling mechanical laminates.

Coupling coefficient $\left(e_{N V}, e_{M V}\right)$. For a given thickness of the piezoelectric layers, the coupling coefficients predicted by ND and NS are independent of the thickness of the elastic layer. On the other hand, the NSR model accounts for a dependence on the thickness ratio $\eta$. Moreover, the ND model sensibly overestimates $(\sim+30 \%)$ the coupling coefficient in comparison to the NS and NSR models. To give an illustrative explication of the underlying phenomena, let us consider the case of the in-phase connected beam and let us write the $e_{N V}$ coefficient as the induced electric charge per unit length for a given axis extension $u^{\prime}=S_{11}$, under the condition of null axis bending and null applied voltage. For a plane-stress state $\left(T_{33}=0\right)$, we find

$$
e_{N V}=\left.\frac{q}{S_{11}}\right|_{w^{\prime \prime}=V=0}=2 a \tilde{e}_{31}\left(1+S_{22} / S_{11}\right) .
$$

In the ND model, since $S_{22}^{(\mathrm{ND})}$ is forced to be zero, the coupling coefficient is given by

$$
e_{N V}^{(\mathrm{ND})}=2 a \tilde{e}_{31} \text {. }
$$

On the other hand, in the NS model, since the transversal stress $T_{22}$ is assumed to be zero, we find

$$
S_{22}^{(\mathrm{NS})}=-\tilde{v}^{\mathrm{E}} S_{11}, \quad e_{N V}^{(\mathrm{NS})}=2 a \tilde{e}_{31}\left(1-\tilde{v}^{\mathrm{E}}\right),
$$

where $\tilde{v}^{\mathrm{E}}$ is the in-plane Poisson coefficient of the piezoelectric material for $T_{33}=0$ and $E_{3}=0$. Hence, for positive Poisson coefficients, $e_{N V}^{(\mathrm{NS})}<e_{N V}^{(\mathrm{ND})}$. In the NSR case, the transverse deformations of the piezoelectric layers are influenced also by the deformation of the elastic layer: if the Poisson ratio $\tilde{v}$ of the elastic layer is greater that the one of the piezoelectric layer (as in the numerical case we considered), then the elastic layer will induce a stronger shrinking for a given axial extension. Hence, for $\tilde{v}^{\mathrm{E}}<\tilde{v}$,

$$
S_{22}^{(\mathrm{NSR})}<S_{22}^{(\mathrm{NS})}, \quad e_{N V}^{(\mathrm{NSR})}<e_{N V}^{(\mathrm{NS})} .
$$


On the contrary, if $\tilde{v}<\tilde{v}^{\mathrm{E}}$ then $e_{N V}^{(\mathrm{NSR})}>e_{N V}^{(\mathrm{NS})}$. The relations above can be verified also by analyzing the ratio between the analytical expressions furnished in Table 2. Such a difference between the coupling coefficient of the NSR and NS model is small because exclusively due to the difference in the Poisson ratio of different layers, which is usually small. In the case of counter-phase connected piezoelectric layers, the underlying phenomena are essentially the same, but some further complications arise because of the though-thethickness linear contributions to the stress and strain which are associated with the beam flexion. As for the equivalent capacitance, for $\eta=0$ also the coupling coefficients of the in-phase connected beam given by the NS model and the NSR model coincide. Indeed, in this case the beam is completely equivalent to a single layer piezoelectric beam and the two conditions (NS) and (NSR) are equivalent. This is not the case for the counter-phase connection. In this case, when $\eta=0$, the sandwich beam reduces to a bimorph piezoelectric pair, where transverse interactions between different layers are important.

\subsubsection{Two-layered beam}

For the asymmetric layer configuration, the potential difference between the electrodes of the piezoelectric layers is intrinsically coupled both to the beam extension and bending, which, in general, are also mechanically coupled to each other. We report in the following table the constitutive coefficients appearing in the unconstrained beam constitutive equations (41), evaluated in a reference frame positioned as in Fig. 3.

$$
\begin{aligned}
& k_{N u}=k_{N u}^{(\mathrm{ND})}=a h_{\mathrm{p}} \tilde{c}_{11}^{\mathrm{E}}\left(1+\eta \tilde{\beta}_{11}\right), \\
& k_{N w}=k_{N w}^{(\mathrm{ND})}=a h_{\mathrm{p}}^{2} \tilde{c}_{11}^{\mathrm{E}} \frac{(1+\eta)}{2}, \\
& k_{M w}=k_{M w}^{(\mathrm{ND})}=\tilde{c}_{11}^{\mathrm{E}} J_{2}\left(3(1+\eta)^{2}+1+\tilde{\gamma}_{11}+\eta^{3} \tilde{\beta}_{11}\right), \\
& e_{N V}=e_{N V}^{(\mathrm{ND})}=a \tilde{e}_{31}, \\
& \epsilon_{q V}=\epsilon_{q V}^{(\mathrm{ND})}=a \tilde{\epsilon}_{33}^{\mathrm{S}} / h_{\mathrm{p}}, \\
& e_{M V}=e_{M V}^{(\mathrm{ND})}=a \tilde{e}_{31} \bar{z}_{2}, \\
& k_{N \lambda}=A_{2} \tilde{c}_{12}^{\mathrm{E}}\left(1+\frac{A_{1} \tilde{c}_{12}}{A_{2} \tilde{c}_{12}^{\mathrm{E}}}\right), \\
& k_{N \mu}=a h_{\mathrm{p}}^{2} \tilde{c}_{12}^{\mathrm{E}} \frac{(1+\eta)}{2}, \\
& k_{M \mu}=\tilde{c}_{12}^{\mathrm{E}} J_{2}\left(3(1+\eta)^{2}+1+\tilde{\gamma}_{11}+\eta^{3} \tilde{\beta}_{12}\right) .
\end{aligned}
$$

As the explicit evaluation of the constitutive coefficients appearing in Eq. (48) becomes rather cumbersome due to the presence of mixed extension and bending stiffness, we limit ourselves to showing some plots for these coefficients obtained in the case of the material properties specified by Table 1. In Fig. 5, the ratio between the constitutive coefficients of the NS and NSR models to those of the ND model are reported. In this case, the one related to the mechanical coupling between extension and bending $\left(k_{N w}\right)$ is not vanishing. Comments similar to those detailed for the three-layered beam hold also in this case, and an analogous interpretation of the dependence on the thickness ratio between the piezoelectric and the elastic layer can be drawn.

\subsection{Comparison of $3 D$ fields underlying the ND, NS and NSR models}

The kinematical state of the three beam models above (ND, NS and NSR) is specified through the fields $(u, w, V)$. They determine beam axis extension, bending, and electric potential, respectively. For a given 

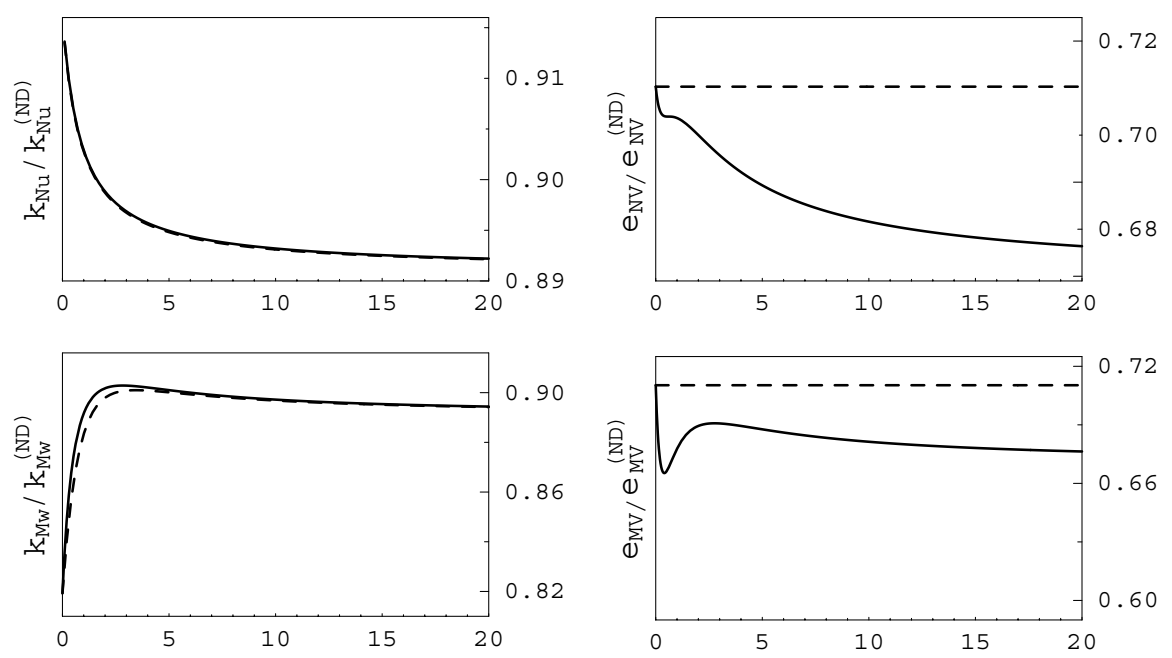

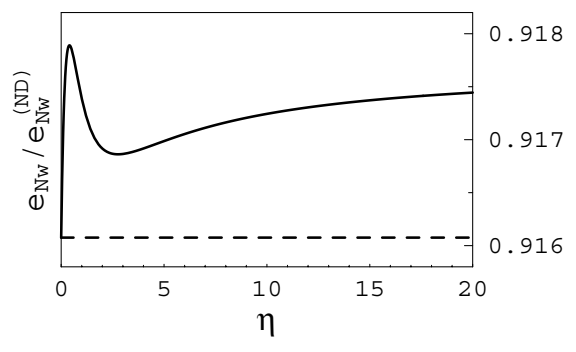

(a)

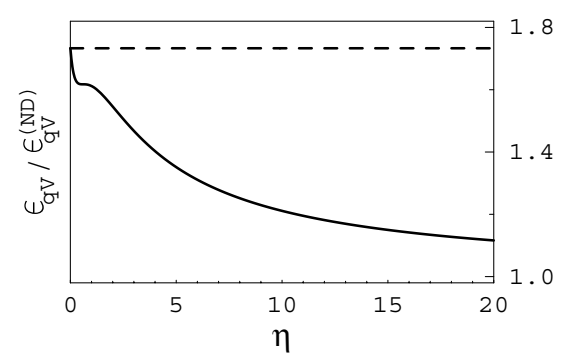

(b)

Fig. 5. Beam constitutive coefficients for two-layered piezoelectric beam as a function of the thickness ratio $\eta=h_{\mathrm{b}} / h_{\mathrm{p}}$. The continuous lines refer to NSR model, the dashed lines to the NS model. The constitutive coefficients are reported as ratios with respect to those estimated by the ND model.

physical situation, their axial distribution is determined as a solution of the associated 1D boundary value problem, consisting of balance equations (32), suitable boundary conditions, and the specific form of the constitutive relations (i.e. Eqs. (59) for the ND model, Eqs. (58) for the NS model and Eqs. (48) for the NSR model). Each model associates given beam generalized deformations $\left(u^{\prime}, w^{\prime \prime}, V\right)$ to different distribution of the three-dimensional state fields $(\mathbf{T}, \mathbf{D}, \overline{\mathbf{S}}, \overline{\mathbf{E}})$. Such distributions are determined as follows: (i) generalized stresses $\left(\sigma_{\alpha, i}, \zeta_{\alpha, i}, \mathrm{D}_{3, i}\right)$ are found by local constitutive equations in the form (36) or (56); (ii) the three-dimensional stress state (T, D) is obtained from the latter through Eqs. (11) and (12); (iii) finally, the $3 \mathrm{D}$ generalized deformations can be found through 3D constitutive equations in the form (2e). Here and henceforth, we will refer to the 3D deformations and electric filed found in this way as constitutive induced deformation and electric field $(\overline{\mathbf{S}}, \overline{\mathbf{E}})$. Indeed, they are the deformation and electric field determined by the stress and electric displacement $(\mathbf{T}, \mathbf{D})$ through constitutive equations (2e). As a peculiarity of the reduced model deduced by a mixed variational formulation, in general they differ from the mechanical strain $\mathbf{S}$ and the electric field $\mathbf{E}$ compatible with the kinematical hypotheses (9) and (10). The $(\overline{\mathbf{S}}, \overline{\mathbf{E}})$ fields are those used to evaluate the internal energy (3).

In the following, the distribution of the 3D state fields associated with the proposed NSR model is compared to the ones related to the standard NS and ND models. In particular, the field distributions corresponding to the following beam kinematical states are analyzed: 
- beam extension: $\left(u^{\prime} \rightarrow S_{0}, w^{\prime \prime} \rightarrow 0, V \rightarrow 0\right)$,

- beam bending: $\left(u^{\prime} \rightarrow 0, w^{\prime \prime} \rightarrow S_{0} / z_{0}, V \rightarrow 0\right)$,

- applied voltage $\left(u^{\prime} \rightarrow 0, w^{\prime \prime} \rightarrow 0, V \rightarrow V_{0}\right)$.

The distribution for a generic beam kinematical state $(u, w, V)$ can be regarded as a linear combination of these three situations. In order to deal with non-dimensional deformations, stress, electric fields and electric displacement, the following scaling quantities are introduced: $S_{0}$ (characteristic mechanical deformation), $z_{0}=h_{\mathrm{p}}$ (characteristic thickness dimension), $T_{0}=\tilde{c}_{11}^{\mathrm{E}} S_{0}$ (characteristic mechanical stress), $E_{0}=S_{0} \sqrt{\tilde{c}_{11}^{\mathrm{E}} / \tilde{\varepsilon}_{33}^{\mathrm{S}}}$ (characteristic electric field), $D_{0}=S_{0} \sqrt{\tilde{c}_{11}^{\mathrm{E}} \tilde{\varepsilon}_{33}^{\mathrm{S}}}$ (characteristic electric displacement), and $V_{0}=E_{0} z_{0}$ (characteristic voltage).

The cases of a two-layered beam and a three-layered beam are considered; the thickness ratio between the piezoelectric and the elastic layers $\left(\eta=h_{b} / h_{\mathrm{p}}\right)$ is fixed to 2. For sake of brevity, for the three-layered beam only the more interesting configuration of a counter-phase connection between the piezoelectric layers is considered. In this case, the electric potential across the electrodes is coupled only to beam bending. We report in Figs. 6 and 7 the distribution of the non-zero component of the three-dimensional strain, stress, electric field and electric displacement for imposed beam bending $\left(u^{\prime} \rightarrow 0, w^{\prime \prime} \rightarrow S_{0} / z_{0}, V \rightarrow 0\right)$ and applied voltage $\left(u^{\prime} \rightarrow 0, w^{\prime \prime} \rightarrow 0, V \rightarrow V_{0}\right)$, respectively. For the two-layered beam in Fig. 5, extension, bending, and applied voltage are all coupled, both by mechanical and piezoelectric effects. We report in Fig. 8-10 the corresponding three-dimensional field distribution for imposed beam extension $\left(u^{\prime} \rightarrow S_{0}, w^{\prime \prime} \rightarrow 0, V \rightarrow 0\right)$, bending $\left(u^{\prime} \rightarrow 0, w^{\prime \prime} \rightarrow S_{0} / z_{0}, V \rightarrow 0\right)$, and applied voltage $\left(u^{\prime} \rightarrow 0, w^{\prime \prime} \rightarrow 0\right.$, $V \rightarrow V_{0}$ ), respectively. The distributions associated to the ND, NS and NSR models are compared (dashed,
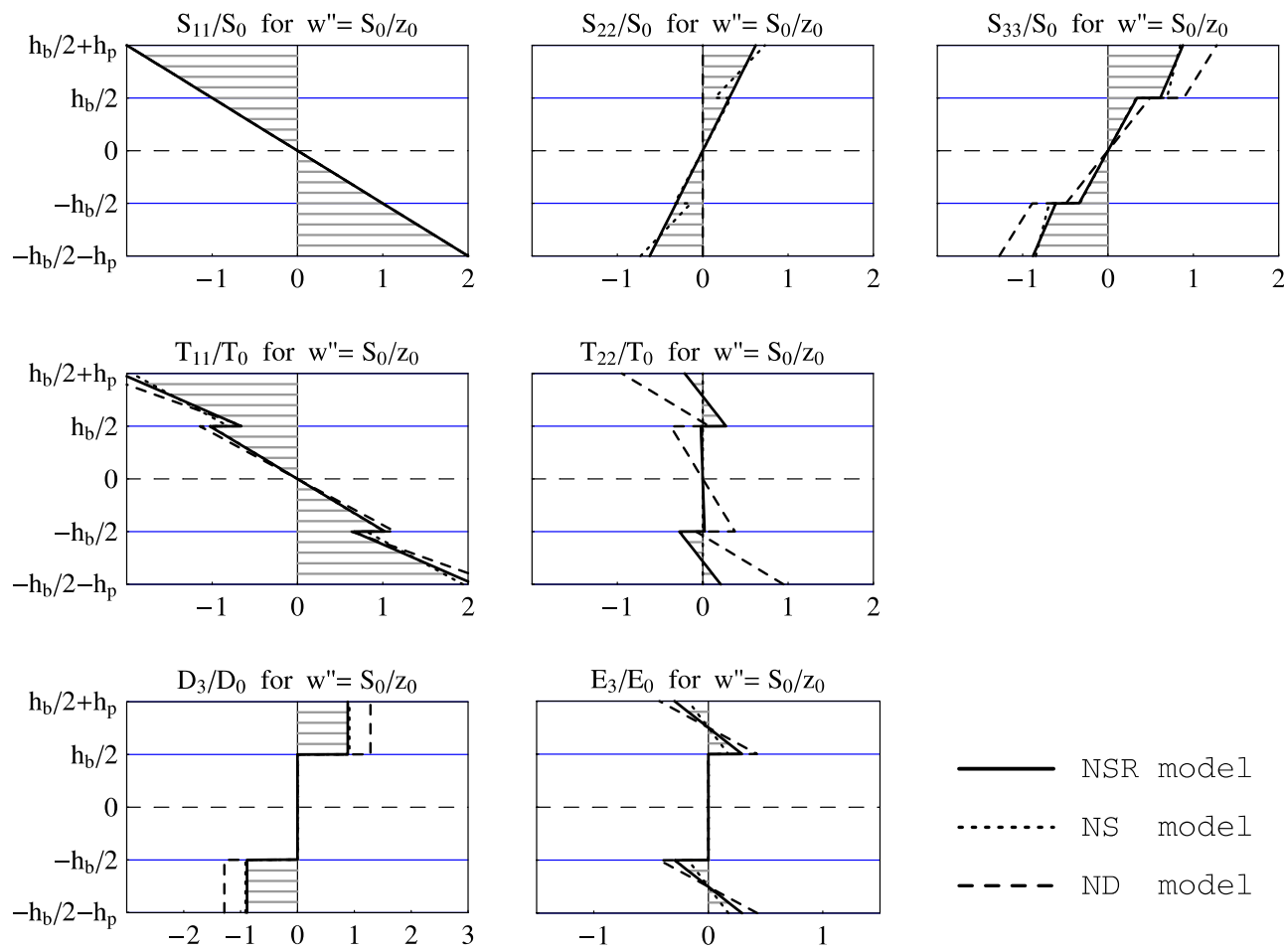

Fig. 6. 3D-field distribution for the three-layered sandwich piezoelectric beam with a counter-phase electric connection and a thickness ratio $\eta=h_{\mathrm{b}} / h_{\mathrm{p}}=2$. A mechanical bending is imposed $\left(u^{\prime} \rightarrow 0, w^{\prime \prime} \rightarrow S_{0} / z_{0}, V \rightarrow 0\right)$. 

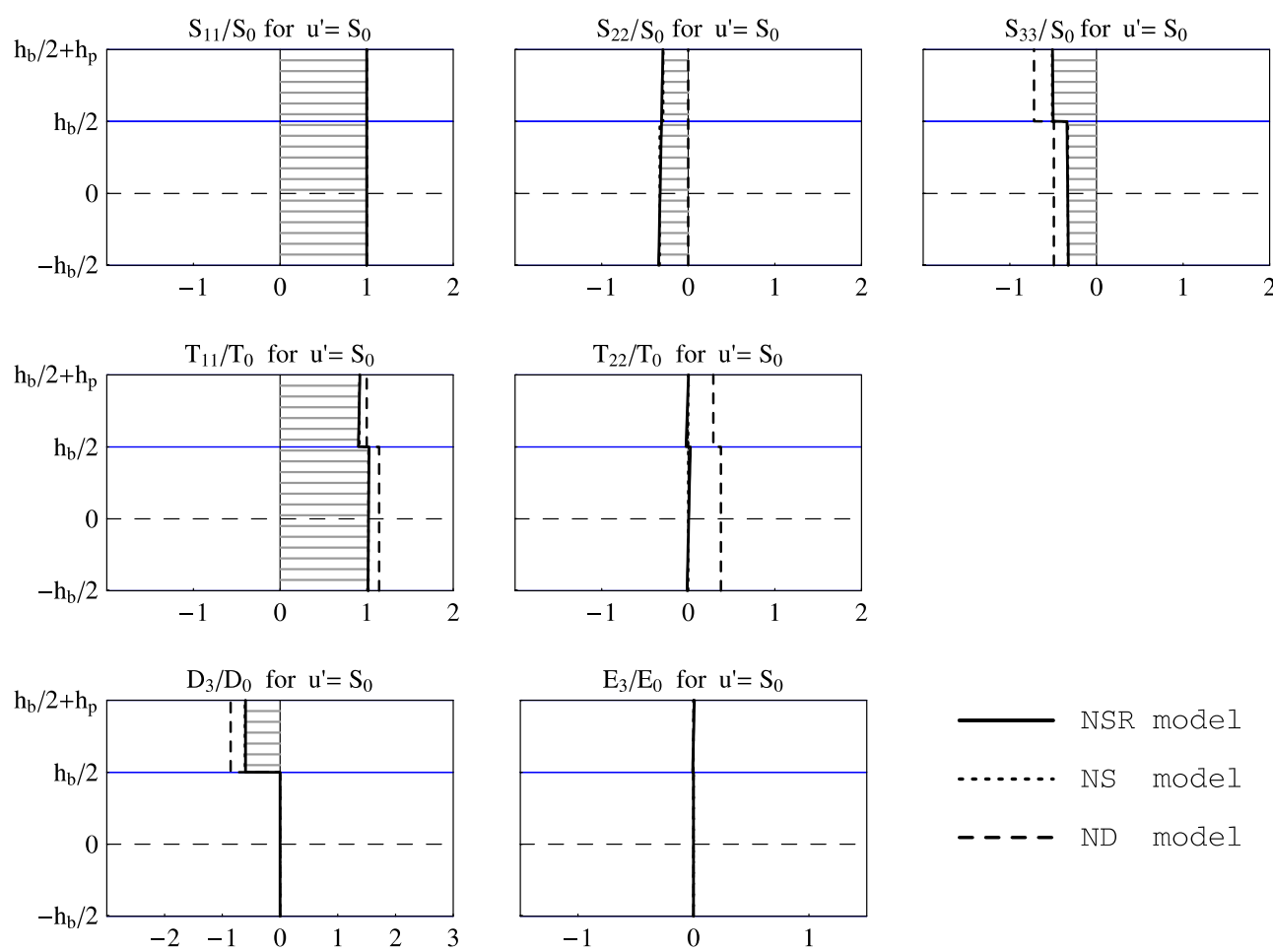

Fig. 8. 3D-field distribution for a two-layered sandwich piezoelectric beam with a thickness ratio $\eta=h_{\mathrm{b}} / h_{\mathrm{p}}=2$. A mechanical extension is imposed $\left(u^{\prime} \rightarrow S_{0}, w^{\prime \prime} \rightarrow 0, V \rightarrow 0\right)$.

(ii) The NS model neglects transverse interactions between different layers and does not take into account the bonding condition in the transverse direction. By condition (14), each layer is left free to deform along the width, independently of the others (see the dotted lines in the $S_{22}$ plots of Figs. 6-10). For example, when a potential difference is applied, while the central elastic layer does not deform along the width, the upper piezoelectric layer extends, the lower one shrinks uniformly, and the bonding condition is not satisfied at the interface (see the $S_{22}$ plot in Fig. 7).

(iii) The ND model neglects transverse sectional deformations and transverse stress transfer between different layers. In each layer transverse stresses are determined by the condition of transverse rigidity, independently of the state of other layers (see the dashed lines of the $T_{22}$ plots in Figs. 6-10). For example, when a potential difference is applied to the three-layered beam (Fig. 7), transverse $T_{22}$ stresses arise in order to assure null transverse deformation; these stresses are present only in the piezoelectric layers and they do not influence the state of the central elastic layer.

(iv) All the three models coherently leave the cross section free to deform in the thickness direction. By assuming vanishing normal stress $T_{33}$ (all three models assume a stress distribution in the form (11)), a sectional distension along the thickness direction is implicitly accounted for (see the layerwise linear distribution of $S_{33}$ in Figs. 7-10). When no forces are prescribed on the beam lateral boundary, each layer either shrinks or extends along the thickness independently of all the others. This component of the deformation is naturally induced both by the standard Poisson effect and piezoelectric coupling (so-called 33 coupling).

(v) All three models coherently account for a linear contribution to the electric field in bent piezoelectric layers. Since by hypothesis (12) the electric displacement has been assumed to be layerwise constant, the 

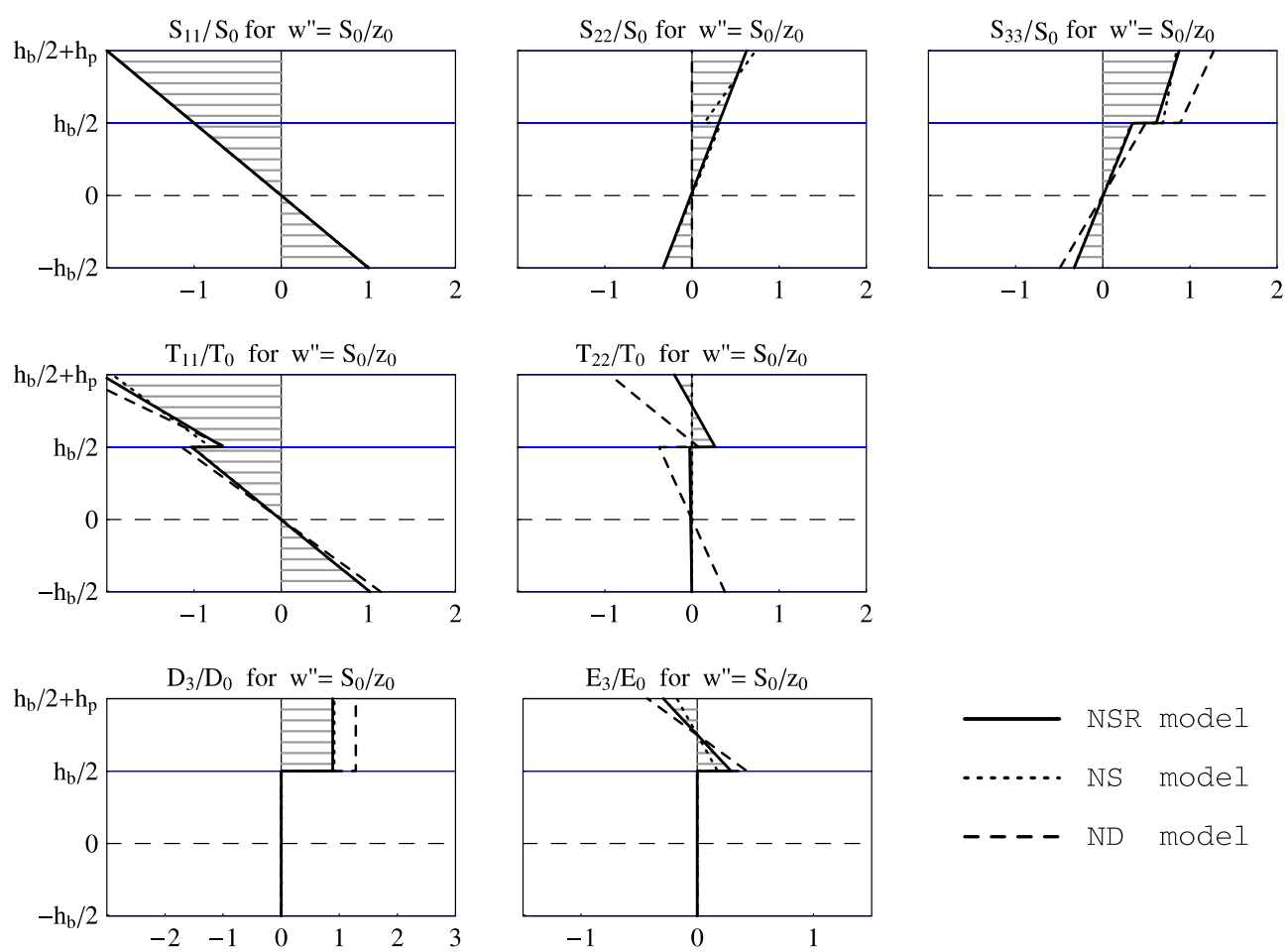

Fig. 9. 3D-field distribution for a two-layered sandwich piezoelectric beam with a thickness ratio $\eta=h_{\mathrm{b}} / h_{\mathrm{p}}=2$. A mechanical bending is imposed $\left(u^{\prime} \rightarrow 0, w^{\prime \prime} \rightarrow S_{0} / z_{0}, V \rightarrow 0\right)$.

linear part of the mechanical deformation constitutively induces a linear contribution to the electric field (quadratic contribution to the electric potential). Hence, when the piezoelectric layers are bent, linear contributions to the electric field are present (see $E_{3}$ plots in Figs. 6-10). As a related effect, when the potential difference applied across the electrodes is null (condition $V=0$ ), piezoelectric layers are characterized by two different mechanical stiffnesses (see local constitutive equations (36) and (56)): the constant contribution to the mechanical deformation is characterized by the mechanical stiffness for a null electric field (superscript E) and the linear contribution by the mechanical stiffness for a null electric displacement (superscript D). Indeed, for $V=0$, the constant part of the electric potential is null, while the linear part is arbitrary; at the same time, the linear part of the electric displacement is null because of hypothesis (12), while the constant one is arbitrary. Hence, the constant part of the mechanical deformation is affected by condition $E_{3}=0$ and the linear one by condition $D_{3}=0$.

(vi) The intensity of the electric displacement for a given electric potential and axial deformation depends on the transverse stress and strain state. It is at a maximum when transverse stresses are null and piezoelectric layers can freely extend or shrink along the width; it is at a minimum when transverse deformations are constrained to be null; it attains a value between these extremes when neither transverse stresses nor transverse deformations are null. As an example, in the $D_{3}$ plots of Figs. 7 and 10, the value of the electric displacement predicted by the NSR model is in between those of the NS and ND models, being null neither transverse stress nor transverse strain. This effect is directly related to the difference in the estimates of the equivalent piezoelectric capacitance per unit length $\varepsilon_{q V}$ in the beam constitutive equations: the NSR model, by catching non-trivial transverse interactions between different layers, furnishes a more realistic estimate of the electric displacement intensity for a given electric potential and axial deformation (and consequently of $\varepsilon_{q V}$ ). 

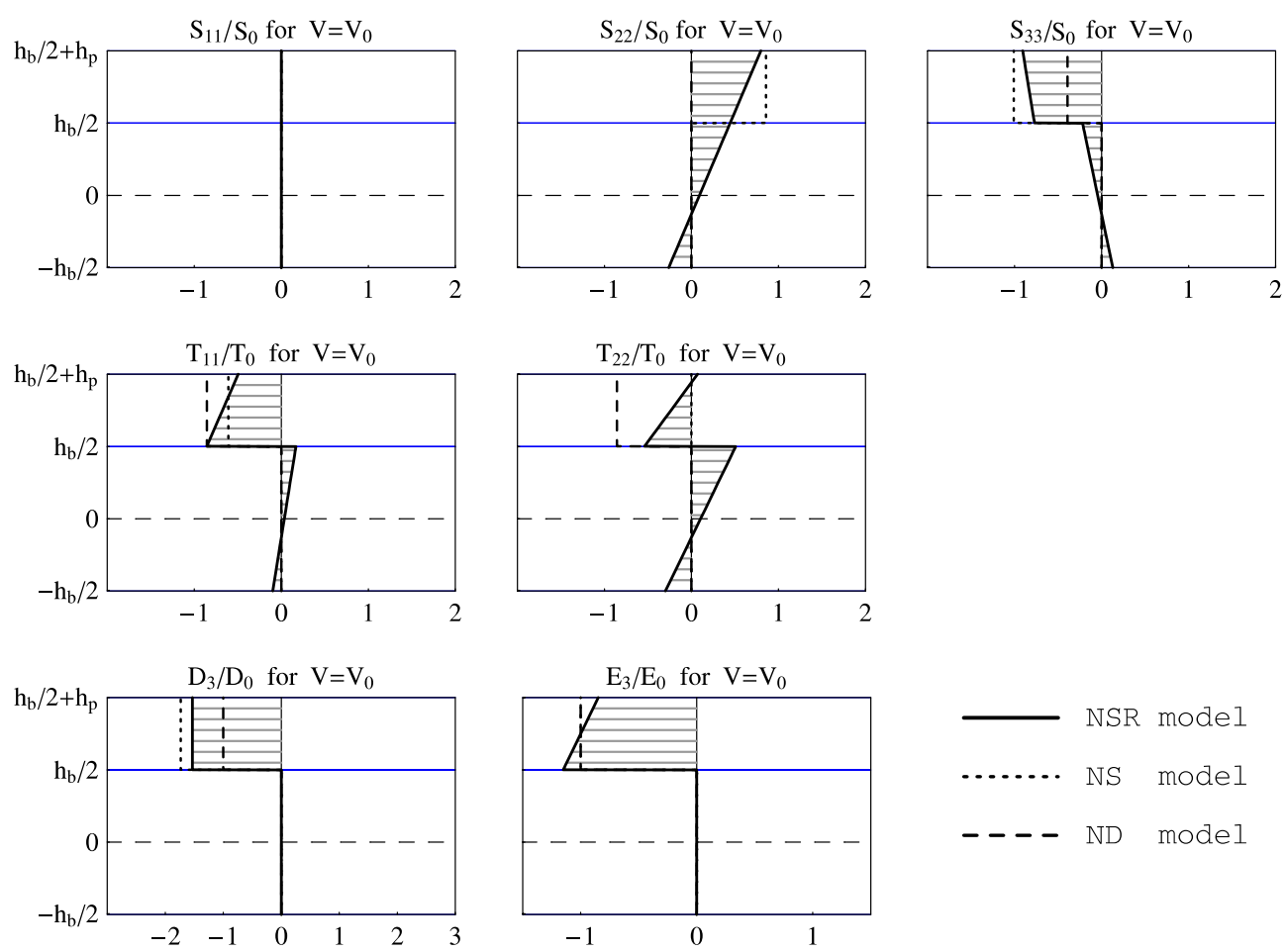

Fig. 10. 3D-field distribution for a two-layered sandwich piezoelectric beam with a thickness ratio $\eta=h_{\mathrm{b}} / h_{\mathrm{p}}=2$. An electric potential is imposed $\left(u^{\prime} \rightarrow 0, w^{\prime \prime} \rightarrow 0, V \rightarrow V_{0}\right)$.

\section{Conclusions}

In this paper, models of layered piezoelectric beams accounting for direct and inverse electromechanical coupling were discussed. An equivalent-single-layer Euler-Bernoulli model was deduced from a threedimensional continuum model by a mixed variational principle. The estimates obtained by simplest modelling approaches are refined without adding additional degrees-of-freedom (such as shear deformations, multilayer kinematics, higher-order electric field distribution, etc.). The model is based on hypotheses (9)-(12) and weak condition (13) on transverse stresses (null transverse stress resultants). Its main peculiarities reside in the following points: (i) to account for transverse interactions between different layers and sectional bending and extension in the transverse direction (thanks to the non-standard weak condition (13) on transverse stress); (ii) to include as state variables all the electric and mechanical state fields and to account for full two-fold piezoelectric coupling between them; (iii) to account for bending of the piezoelectric layers by including coherent distributions of electric field and displacement (by imposing in the mixed variational formulation a layerwise constant distribution of the electric displacement through hypothesis (H4)).

The attention was focused on the influence of hypotheses on transverse normal stress and strain on the deduction of the beam constitutive coefficients from three-dimensional material and geometric properties and on the reconstruction of the through-the-thickness distribution of three-dimensional state fields. It was shown that, differently from models assuming either null transverse deformations or null transverse stress, the model assuming null transverse stress resultants takes into account transverse interactions between different layers and the related non-trivial transverse strain and stress distribution. As main benefit, such a 
model correctly estimates the beam constitutive coefficients relating axial deformations and potential difference across piezoelectric layers to axial stress resultants and stored charge. In particular, one of the main weaknesses shown by standard one-dimensional models is overcome by improving the estimate of equivalent piezoelectric capacitance. Indeed, this property is strongly dependent on the assumed distribution of transverse strain and stress.

The theoretical analysis developed in the present paper was motivated by some experimental falsifications of the ND and NS models. More precisely, a preliminary set of measurements which we performed indicated that the piezoelectric capacitance of layered beams were not correctly predicted. As a natural extension of the present work, future investigations will involve the experimental validation of the presented NSR model and its estimates of electromechanical constitutive properties. To this end, experimental setups aimed at the characterization of the electrical and mechanical properties of layered piezoelectric beams must be performed. On the other hand, the most prominent theoretical refinement to be included in the model is the description of non-perfect bonding between different layers and the main phenomena related to the presence of the bonding layer.

\section{Appendix A. Constitutive coefficients}

\section{A.1. Local constitutive equations}

The constitutive coefficients appearing in the form (36) of the local constitutive equations are expressed as a function of $\left(s_{11}^{\mathrm{E}}, s_{12}^{\mathrm{E}}, d_{31}, \beta_{33}^{\mathrm{T}}\right)$ as follows. They correspond to the constitutive coefficients of the 3D piezoelectric constitutive equations in the so-called $\mathbf{S}-\mathbf{E}$ form for a plane-stress condition in the $\mathbf{e}_{1}-\mathbf{e}_{2}$ plane and a uniaxial electric displacement along $\mathbf{e}_{3}$.

$$
\begin{aligned}
& \tilde{c}_{11}^{\mathrm{E}}=\frac{s_{11}^{\mathrm{E}}}{\left(s_{11}^{\mathrm{E}}\right)^{2}-\left(s_{12}^{\mathrm{E}}\right)^{2}}, \\
& \tilde{c}_{12}^{\mathrm{E}}=-\frac{s_{12}^{\mathrm{E}}}{\left(s_{11}^{\mathrm{E}}\right)^{2}-\left(s_{12}^{\mathrm{E}}\right)^{2}}, \\
& \tilde{e}_{31}=-\frac{d_{31}}{s_{11}^{\mathrm{E}}+s_{12}^{\mathrm{E}}}, \\
& \tilde{\epsilon}_{33}^{\mathrm{S}}=\frac{1}{\beta_{33}^{\mathrm{T}}}-2 \frac{d_{31}^{2}}{s_{11}^{\mathrm{E}}+s_{12}^{\mathrm{E}}}, \\
& \tilde{c}_{11}^{\mathrm{D}}=\tilde{c}_{11}^{\mathrm{E}}+\tilde{e}_{31}^{2} / \tilde{\varepsilon}_{33}^{\mathrm{S}}, \\
& \tilde{c}_{12}^{\mathrm{D}}=\tilde{c}_{12}^{\mathrm{E}}+\tilde{e}_{31}^{2} / \tilde{\varepsilon}_{33}^{\mathrm{S}} .
\end{aligned}
$$

On the other hand, in Eqs. (56) the constitutive coefficients for an uniaxial stress along $\mathbf{e}_{1}$ and an uniaxial electric displacement along $\mathbf{e}_{3}$ appear. The are given by

$$
\begin{aligned}
& \hat{c}_{11}^{\mathrm{E}}=\tilde{c}_{11}^{\mathrm{E}}\left(1-\left(\tilde{c}_{12}^{\mathrm{E}} / \tilde{c}_{11}^{\mathrm{E}}\right)^{2}\right)=\frac{1}{s_{11}^{\mathrm{E}}}, \\
& \hat{e}_{31}=\tilde{e}_{31}\left(1-\tilde{c}_{12}^{\mathrm{E}} / \tilde{c}_{11}^{\mathrm{E}}\right)=-\frac{d_{31}}{s_{11}^{\mathrm{E}}},
\end{aligned}
$$




$$
\begin{aligned}
& \hat{\epsilon}_{33}^{\mathrm{S}}=\tilde{\epsilon}_{33}^{\mathrm{S}}\left(1+\frac{\tilde{e}_{31}^{2}}{\tilde{c}_{11}^{\mathrm{E}} \tilde{\epsilon}_{33}^{\mathrm{S}}}\right)=\frac{1}{\beta_{33}^{\mathrm{T}}}-\frac{d_{31}^{2}}{s_{11}^{\mathrm{E}}}, \\
& \hat{c}_{11}^{\mathrm{D}}=\tilde{c}_{11}^{\mathrm{D}}\left(1-\left(\tilde{c}_{12}^{\mathrm{D}} / \tilde{c}_{11}^{\mathrm{D}}\right)^{2}\right)=\hat{c}_{11}^{\mathrm{E}}+\hat{e}_{31}^{2} / \hat{\epsilon}_{33}^{\mathrm{S}} .
\end{aligned}
$$

For the elastic layers, the coefficients $\left(\tilde{c}_{11}, \tilde{c}_{12}\right)$ and $\hat{c}_{11}$ are found by expressions analog to (A.1) and (A.2) where piezoelectric compliances must be substituted by the corresponding elastic compliances.

\section{A.2. Global constitutive equations}

The constitutive coefficients appearing in Eq. (41) are found by substituting the local constitutive equations (36) and the relations of kinematical compatibility in the definition of the stress resultants (33) and (37). The following expressions are found:

$$
\begin{aligned}
& k_{N u}=k_{N u}^{(\mathrm{ND})}=\sum_{i \in \mathscr{G}_{\mathrm{p}}} A_{i} \tilde{c}_{11}^{\mathrm{E}}+\sum_{i \in \mathscr{I}_{\mathrm{e}}} A_{i} \tilde{c}_{11}, \\
& k_{N w}=k_{N w}^{(\mathrm{ND})}=\sum_{i \in \mathscr{I}_{\mathrm{p}}} A_{i} \bar{z}_{i} \tilde{c}_{11}^{\mathrm{E}}+\sum_{i \in \mathscr{G}_{\mathrm{e}}} A_{i} \bar{z}_{i} \tilde{c}_{11}, \\
& k_{M w}=k_{M w}^{(\mathrm{ND})}=\sum_{i \in \mathscr{I}_{\mathrm{p}}}\left(A_{i} \bar{z}_{i}^{2} \tilde{c}_{11}^{\mathrm{E}}+\tilde{c}_{11}^{\mathrm{D}} J_{i}\right)+\sum_{i \in \mathscr{I}_{\mathrm{e}}} \tilde{c}_{11}\left(A_{i} \bar{z}_{i}^{2}+J_{i}\right), \\
& e_{N V}=e_{N V}^{(\mathrm{ND})}=\sum_{i \in \mathscr{I}_{\mathrm{p}}} A_{i} \omega_{i} \tilde{e}_{31} / h_{i}=\sum_{i \in \mathscr{I}_{\mathrm{p}}} a_{i} \omega_{i} \tilde{e}_{31}, \\
& e_{M V}=e_{M V}^{(\mathrm{ND})}=\sum_{i \in \mathscr{I}_{\mathrm{p}}} \omega_{i} \tilde{e}_{31} \bar{z}_{i} a_{i}, \\
& \epsilon_{q V}=\epsilon_{q V}^{(\mathrm{ND})}=\sum_{i \in \mathscr{I}_{\mathrm{p}}} a_{i} \tilde{\epsilon}_{33}^{\mathrm{S}} / h_{i}, \\
& k_{N \mu}=\sum_{i \in \mathscr{I}_{\mathrm{p}}} A_{i} \tilde{c}_{12}^{\mathrm{E}}+\sum_{i \in \mathscr{I}_{\mathrm{p}}} A_{i} \bar{z}_{i} \tilde{c}_{i 2}^{\mathrm{E}}+\sum_{i \in \mathscr{I}_{\mathrm{e}}} \tilde{c}_{12} A_{i} \bar{z}_{i} \tilde{c}_{12}, \\
& k_{M \mu}=\sum_{i \in \mathscr{I}_{\mathrm{p}}}\left(A_{i} \bar{z}_{i}^{2} \tilde{c}_{12}^{\mathrm{E}}+J_{i} \tilde{c}_{12}^{\mathrm{D}}\right)+\sum_{i \in \mathscr{G}_{\mathrm{e}}} \tilde{c}_{12}\left(A_{i} \bar{z}_{i}^{2}+J_{i}\right) .
\end{aligned}
$$

Similarly, the constitutive constants appearing in Eq. (58) are found by the local constitutive equations (56). They are given by

$$
\begin{aligned}
k_{N u}^{(\mathrm{NS})} & =\sum_{i \in \mathscr{I}_{\mathrm{p}}} A_{i} \hat{c}_{11}^{\mathrm{E}}+\sum_{i \in \mathscr{I}_{\mathrm{e}}} A_{i} \hat{c}_{11}, \\
k_{N w}^{(\mathrm{NS})} & =\sum_{i \in \mathscr{I}_{\mathrm{p}}} \bar{z}_{i} A_{i} \hat{c}_{11}^{\mathrm{E}}+\sum_{i \in \mathscr{I}_{\mathrm{e}}} \bar{z}_{i} A_{i} \hat{c}_{11}, \\
k_{M w}^{(\mathrm{NS})} & =\sum_{i \in \mathscr{I}_{\mathrm{p}}}\left(\hat{c}_{11}^{\mathrm{D}} J_{i}+\bar{z}_{i}^{2} A_{i} \hat{c}_{11}^{\mathrm{E}}\right)+\sum_{i \in \mathscr{I}_{\mathrm{e}}} \hat{c}_{11}\left(J_{i}+\bar{z}_{i}^{2} A_{i}\right), \\
e_{M V}^{(\mathrm{NS})} & =\sum_{i \in \mathscr{I}_{\mathrm{p}}} \bar{z}_{i} A_{i} \hat{e}_{31} \omega_{i} / h_{i}, \\
e_{N V}^{(\mathrm{NS})} & =\sum_{i \in \mathscr{I}_{\mathrm{p}}} A_{i} \hat{e}_{31} \omega_{i} / h_{i}, \\
\epsilon_{q V}^{(\mathrm{NS})} & =\sum_{i \in \mathscr{I}_{\mathrm{p}}} a_{i} / h_{i} \hat{\epsilon}_{33}^{\mathrm{S}} .
\end{aligned}
$$




\section{References}

Alzahrani, B.A., Alghamdi, A.A.A., 2003. Review of the mechanics of materials models for one-dimensional surface-bonded piezoelectric actuators. Smart Mater. Struct. 12, N1-N4.

Anderson, E.H., Hagood, N.W, 1994. Simultaneous piezoelectric sensing/actuator: analysis and application to controlled structures. J. Sound Vibrat. 174 (5), 617-639.

Batra, R.C., Vidoli, S., 2002. Higher-order piezoelectric plate theory derived from a three-dimensional variational principle. AIAA J. 40 (1), 91-104.

Beckert, W., Pfundtner, G., 2002. Analysis of the deformational behaviour of a bimorph configuration with piezoelectric actuation. Smart Mater. Struct. 11, 599-609.

Chee, C.Y.K., Tong, L.S., Grant, P., 1998. Review on the modelling of piezoelectric sensors and actuators incorporated in intelligent structures. J. Intell. Mater. Syst. Struct. 9 (1), 3-19.

Chopra, I., 2002. Review of state of art of smart structures and integrated systems. AIAA J. 40 (11), 2145-2187.

Crawley, E.F., de Luis, J., 1987. Use of piezoelectric actuators as elements of intelligent structures. AIAA J. 25, 1375-1385.

Crawley, E.F., Anderson, E.H., 1990. Detailed models of piezoceramic actuation of beams. J. Intell. Mater. Syst. Struct. 1, 12-25.

Eringen, A., Maugin, G.A., 1990. Electrodynamics of Continua I. Springer, New York.

Gopinathan, S.V., Varadan, V.V., Varadan, V.K., 2000. A review and critique of theories for piezoelectric laminates. Smart Mater. Struct. 9, 24-48.

Hagood, N.W., Von Flotow, A., 1991. Damping of structural vibrations with piezoelectric materials and passive electrical networks. J. Sound Vibrat. 146, 143-168.

He, J.-H., 2000. Generalized Hellinger-Reissner principle. J. Appl. Mech. 67, 326-331.

Hong, C.H., Chopra, I., 1999. Modeling and validation of induced strain actuation of composite coupled plates. AIAA J. $37,372-377$.

Ikeda, T., 1990. Fundamentals of Piezoelectricity. Oxford University Press, New York.

Park, C., Walz, C., Chopra, I., 1996. Bending and torsion models of beams with induced-strain actuators. Smart Mater. Struct. 5, 98-113.

Rao, S.S., Sunar, M., 1994. Piezoelectricity and its use in disturbance sensing and control of flexible structures: a survey. Appl. Mech. Rev. 47, 113-123.

Saravanos, D.A., Heyliger, P.R., 1999. Mechanics and computational models for laminated piezoelectric beams, plates, and shells. Appl. Mech. Rev. 52, 305-320.

Sze, K.Y., Yang, X.-M., Fan, H., in press. Electric assumptions for piezoelectric laminate analysis. Int. J. Solids Struct. 41, $2363-2382$.

Tani, J., Takagi, T., Qui, J., 1998. Intelligent material systems: application of functional materials. Appl. Mech. Rev. 51, 505-521.

Teresi, L., Tiero, A., 1997. On variational approaches to plate model. Meccanica 32, 143-156.

Washizu, K., 1982. Variational methods in Elasticity and Plasticity. Pergamon Press, New York.

Yang, J.S., Batra, R.C., 1995. Mixed variational principles in nonlinear piezoelectricity. Int. J. Nonlinear Mech. 30 (5), $719-726$. 